\title{
NEW ZEALAND \\ NATURAL RADIOCARBON MEASUREMENTS I-V
}

\author{
T. L. GRANT-TAYLOR and T. A. RAFTER
}

New Zealand Geological Survey and Institute of Nuclear Science, Department of Scientific and Industrial Research, Lower Hutt, New Zealand

This list comprises dating determinations of the New Zealand Radiocarbon Laboratory.

All dates listed herein were published previously (NZ-1-78 in Fergusson and Rafter, 1953, 1955, 1957); NZ-79-264 in Fergusson and Rafter, 1959); NZ-265 in Grant-Taylor and Rafter, 1962.

NZ-1-4 were counted by the solid-carbon method and NZ-10 onward by gas-counting methods; details of the methods are given in Rafter (1953, 1955a) and Fergusson and Rafter (1953, 1955). Where possible, allowance has been made for biological fractionation effects by use of secondary standards (Rafter, 1953b). The accuracy of the methods is discussed by McCallum (1955) .

The ages reported have a correction of $120 \mathrm{yr}$ for industrial carbon in material that lived on land and of $100 \mathrm{yr}$ in material that lived in shallow seas. Material from deep oceanic environments and from Antarctica has been excluded from this list, even though it may have appeared previously (Fergusson and Rafter, 1957, 1959). It has become clear that material in the deep oceans is formed with a $\mathrm{C}^{14}$ content that varies considerably, and "dates" on such material have an initial uncertainty that may exceed $3000 \mathrm{yr}$. The significance that can be attached to such dates is no more than an upper limit of age.

In the list, grid references are given for most New Zealand localities in terms of the National 1000-yd grids published in NZMS-1 maps. The age in all cases is given in years before A.D. 1950, in terms of the old half life of $\mathrm{C}^{14}$ of $5568 \pm 30 \mathrm{yr}$.

We have adhered as closely as possible to the data in the published New Zealand lists 1 through 3 . Some descriptions have been expanded.

In conformity with the decision of the Cambridge conference 1963 dates in terms of the present calendar are included. However a recent reassessment of the half life of $\mathrm{C}^{14}$ suggests the presence of a $5 \%$ error from this source. Also work done by various laboratories, de Vries, 1958, Willis et al., 1960 and Jansen, 1962 suggests an additional variation of irregular nature and unknown origin. The magnitude of this variation as determined by three of the four laboratories appears to be similar, although the absolute amount of the variation at a given time may vary. The maximum deviation as determined by tree ring counts is ca. $50 \%$ of the $\mathrm{C}^{14}$ age.

\section{SAMPLE DESCRIPTIONS}

\section{Taupo series}

Dates a violent rhyolitic eruption in NW of Lake Taupo. 


\section{NZ-1. Taupo, N. Z.}

$$
\begin{array}{r}
1820 \\
\text { A.D. } 130
\end{array}
$$

Charcoal from coarse Taupo pumice, close to the main Taupo-Rotorua Road ca. $0.5 \mathrm{mi} \mathrm{S}$ of burnt Forest Products Plantations $\left(38^{\circ} 36^{\prime} \mathrm{S}\right.$ Lat, $176^{\circ}$ 09' E Long), grid ref. N94/624492. Coll. by I. L. Baumgart, N. Z. Soil Bur., Wellington. Comment: dates the eruption of pumice blocks that carbonized the wood.

\section{NZ-2. Taupo, N. Z.}

$3440 \pm 70$

$$
1490 \text { в.с. }
$$

Charcoal from fossil soil, in quarry face, ca. $1 \mathrm{mi}$ SE of Terrace Hotel on the Napier Taupo Rd. ( $38^{\circ} 43^{\prime}$ S Lat, $176^{\circ} 07^{\prime}$ E Long). Coll. by I. L. Baumgart. Comment: date places a younger limit on underlying Waimahia lapilli member.

\section{NZ-3. Taupo, N. Z.}

$1640 \pm 70$

Charcoal from surface of carbonized log in coarse Taupo pumice, depth $3 \mathrm{ft}$ below top of pumice, N. Z. Electricity Dept. road 1/3 mi N of Wanganui River ( $39^{\circ} 01^{\prime}$ S Lat, $175^{\circ} 37^{\prime}$ E Long). Coll. by N. H. Taylor, N. Z. Soil Bur.

\section{NZ-4. Taupo, N. Z.}

$1920 \pm 150$

A.D. 30

Charcoal from water-sorted Taupo pumice near Desert Rd., S bank of Waihohonu Stream, $18 \mathrm{mi} \mathrm{N}$ of Waiouru ( $39^{\circ} 13^{\prime} \mathrm{S}$ Lat, $175^{\circ} 44^{\prime} \mathrm{E}$ Long), N112/223734. Coll. by N. H. Taylor.

\section{NZ-5. Rangitawa Stream near Kakiriki}

Wood from Ohakeo alluvium, ca. $1 \mathrm{ft}$ above base of alluvium, which rests unconformably on Castlecliffian sandy mudstone, on $\mathrm{S}$ bank of Rangitawa Stream across railway from McLennan's gate $\left(40^{\circ} 08^{\prime} \mathrm{S}\right.$ Lat, $175^{\circ} 27^{\prime} \mathrm{E}$ Long), N143/953626. Coll. by M. T. Te Punge, N. Z. Geol. Surv., Wellington. Comment: sample dates the alluvium, interpreted as younger postglacial valley fill.

\section{NZ-6. Pollen Island, Waitemata Harbor}

Mangrove-swamp peat from top $4 \mathrm{in}$. in layer ca. $2 \mathrm{ft}$ thick, overlain by marine silt, $1 \mathrm{ft}$ thick $\left(36^{\circ} 52^{\prime} \mathrm{S}\right.$ Lat, $174^{\circ} 40^{\prime}$ E Long), N2/185593. Subm. by V. J. Chapman, Auckland Univ. Comment: date places a younger limit on age of formation, which would therefore appear to be no younger than the last interglacial age.

\section{NZ-7. Aramaho, Wanganui, North Island}

$2400 \pm 170$

450 в.c.

Fossil tree trunk, rooted in place, at bottom of Aramaho pumice quarry, remnant of a forest on flats beside Wanganui River that was buried by floods of pumice alluvium $\left(39^{\circ} 53^{\prime} \mathrm{S}\right.$ Lat, $175^{\circ} 06^{\prime} \mathrm{E}$ Long), N138/624917. Coll. by C. A. Fleming, N. Z. Geol. Surv.

\section{NUZ-8. Aramaho, Wanganui, North Island}

Charcoal fragments, depth 2 to $5 \mathrm{ft}$ below surface in Aramaho pumice 
quarry, same locality as NZ-7. Charcoal is believed to have been formed at the time of the pumice eruption and almost immediately brought down to the coast by the Wanganui River. Coll. by C. A. Fleming. Comment: age is similar to NZ-7 and supports the interpretation, but the difference as compared with NZ-1 through NZ-4, (this date list) suggests the possibility of another eruption in the district.

\section{NZ-9. Wanganui Valley, Westland, South Island \\ $930 \pm 150$ \\ A.D. 1020}

Wood, buried in gravel deposit that was deformed by late movement of the Alpine fault, Wanganui Valley, $1.25 \mathrm{mi}$ upstream from road bridge $\left(43^{\circ}\right.$ $10^{\prime}$ S Lat, $170^{\circ} 37^{\prime}$ E Long). Coll. by C. A. Fleming.

\section{NZ-10. Kaingaroa Forest, Rotorua}

$\begin{aligned} 930 & \pm 70 \\ \text { A.D. } & 1020\end{aligned}$

Wood from 10-in. depth below surface, 3 in. above contact with buried soil developed on Taupo volcanic ash, imbedded in and stratigraphically overlain by Kaharoa ash, 4 ft thick, and then by Tarawera ash, 6 in. thick, exposed in cut on Northern Boundary Rd., Kaingaroa Forest (38 $19^{\prime}$ S Lat, $176^{\circ} 44^{\prime}$ E Long), N86/127801. Coll. by I. L. Baumgart. Comment: sample places an older limit on age of Kaharoa eruption and approximately dates the eruption.

\section{NZ-11. Penrose, Auckland}

$9270 \pm 80$

7320 в.с.

Wood from tree trunk underlying basalt flow, in excavation for overhead bridge on the Penrose main-road deviation ( $36^{\circ} 54^{\prime} \mathrm{S}$ Lat, $174^{\circ} 49^{\prime} \mathrm{E}$ Long), N42/330540. Subm. by J. Healy, N. Z. Geol. Surv., Rotorua. Comment: dates one of the younger basalts of the Auckland district.

\section{NZ-12. Napier}

$20,670 \pm 300$

$$
18,720 \text { в.c. }
$$

Wood, $5 \mathrm{ft}$ below surface, in deeply weathered pumiceous clay, 9 in. above unweathered pumice and ash, exposed in excavation for Napier Hospital nurses' home, Hospital Hill, Napier ( $39^{\circ} 29^{\prime} \mathrm{S}$ Lat, $176^{\circ} 54^{\prime}$ E Long), N134/ 317397. Coll. by J. A. Berry, George St., Napier. Comment: sample places a younger limit on deposition of pumice ash and an older limit on deposition of the pumiceous clay, which is loess-like.

\section{NZ-13. Rapahoe, Greymouth, Westland \\ $4720 \pm 70$ \\ 2770 в.с.}

Wood from a large tree trunk, $15 \mathrm{ft}$ above sealevel, imbedded in marine gravel terrace at Rapahoe $\left(42^{\circ} 23^{\prime} \mathrm{S}\right.$ Lat, $171^{\circ} 15^{\prime} \mathrm{E}$ Long), S44//766972. Coll. by R. P. Suggate, N. Z. Geol. Surv., Christchurch. Comment: terrace apparently represents deposition at postglacial sealevel higher than the present one, but tectonic uplift is also probable.

\section{NZ-14. Featherston, Wairarapa}

Wood from peat bed, encountered in boring $150 \pm 5 \mathrm{ft}$ below sealevel, in an area of postglacial aggradation $\left(41^{\circ} 13^{\prime} \mathrm{S}\right.$ Lat, $175^{\circ} 18^{\prime} \mathrm{E}$ Long), N161/ 823303. Coll. by I. Barton, Featherston. Comment: sample places a younger limit on peat bed, which is clearly not postglacial. 


\section{NZ-15. Ohariu Valley, Wellington}

$$
\mathbf{8 4 0} \pm 50
$$

\section{$\mathbf{8 4 0}$}

Wood from layer of driftwood, overlain by carbonaceous blue clay, $2 \mathrm{ft}$ thick, and then by sandy silt, $8 \mathrm{ft}$ thick, making a depositional river terrace that reaches $12 \mathrm{ft}$ above present stream level $\left(41^{\circ} 14^{\prime} \mathrm{S}\right.$ Lat, $174^{\circ} 44^{\prime} \mathrm{E}$ Long), N164/294289. Coll. by J. W. Brodie, N. Z. Oceanog. Inst., Wellington. Comment: sequence records local aggradation followed by downcutting; cause of the events is not certainly known.

\section{NZ-16. Buna Kokodu area, New Guinea $\quad 13,870 \pm 250$}

Wood from tree trunk imbedded in ash of the earliest phase of volcanic activity in the district $\left(9^{\circ} 05^{\prime} \mathrm{S}\right.$ Lat, $148^{\circ} 09^{\prime} \mathrm{E}$ Long $)$, Mt. Lamington, an active volcano in the district, has ash deposits that are little consolidated, and is regarded as having begun activity within the last 20,000 yr. Coll. by C. S. Christian, CSIRO, Canberra, Australia.

\section{NZ-17. Titahi Bay, Wellington}

Wood from submerged forest, exposed between tide levels, Titahi Bay ( $41^{\circ} 06^{\prime} \mathrm{S}$ Lat, $174^{\circ} 50^{\prime} \mathrm{E}$ Long), N160/389441. Bay is backed by a low cliff cut in Pleistocene alluvium; forest bed consists of fossil soil with abundant vegetable remains and stumps in growth position. Flora suggests a climate similar to the present, and hence that the forest is of (last?) interglacial age. Coll. by C. A. Fleming.

\section{NZ-18. Fielding}

Impure lignified peat, underlying topmost sandstone that covers the (very young) Fielding anticline, exposed in Walter Seiferts coal mine, Ranfurly Ave., Fielding $\left(40^{\circ} 13^{\prime} \mathrm{S}\right.$ Lat, $175^{\circ} 33^{\prime} \mathrm{E}$ Long $)$, N144/052521. Coll. by M. T. Te Punga.

\section{NZ-19. Palmerston North}

Well-preserved wood, probably totara (Podocarpus totara), in gray mudstone ca. $30 \mathrm{ft}$ above river level, $0.5 \mathrm{mi}$ upstream from Fitzherbert Bridge, Palmerston $\left(40^{\circ} 23^{\prime} \mathrm{S}\right.$ Lat, $175^{\circ} 38^{\prime}$ E Long), N149/123327. Coll. by M. T. Te Punga.

\section{NZ-20. Rangitikei Valley}

Wood from $\log$ with annual rings in Rangitawa Stream $\left(40^{\circ} 08^{\prime} \mathrm{S}\right.$ Lat, $175^{\circ} 28^{\prime}$ E Long), N143/964624. Sample, imbedded in sediments with upper Castlecliffian fossils, was bored by marine lamellibranchs. Collected from beds $25 \mathrm{ft}$ below the unconformity that marks base of Ohakea alluvium. Coll. by M. T. Te Punga and A. L. Bloom.

\section{NZ-21. Palliser Bay}

Well-preserved wood from Pounui formation, in sandy mudstone ca. $20 \mathrm{ft}$ above Wharepapa Stream $\left(41^{\circ} 22^{\prime} \mathrm{S}\right.$ Lat, $175^{\circ} 05^{\prime} \mathrm{E}$ Long $)$, N165/615117. Coll. by A. L. Bloom, M. T. Te Punga, and C. A. Cotton. 
Wood from mudstone lens in Otaki sandstone, overlain by terrace gravel of early last glaciation, Waikanae River, $0.25 \mathrm{mi}$ downstream from railway bridge $\left(40^{\circ} 53^{\prime} \mathrm{S}\right.$ Lat, $175^{\circ} 03^{\prime}$ E Long), N156/590710. Coll. by M. T. Te Punga.

NZ-23. Palliser Bay

Wood in "coverhead," 80 to $90 \mathrm{ft}$ thick, $60 \mathrm{ft}$ above base of "coverhead," in beach cliff 200 yd E of Lake Ferry Hotel (41 $24^{\circ} \mathrm{S}$ Lat, $175^{\circ} 09^{\prime} \mathrm{E}$ Long), N165/675087. Coll. by R. L. Kite, Victoria Univ., Wellington. Comment: "coverhead" is considered to have been deposited during last interglacial.

\section{NZ-24. Cape Palliser}

Well-preserved wood, $5 \mathrm{ft}$ above base of gravel composing emerged coastal plain that fringes part of $\mathrm{E}$ side of Palliser Bay, near Waitarangi wool-shed on road to lighthouse (41 ${ }^{\circ} 28^{\prime} \mathrm{S}$ Lat, $175^{\circ} 138^{\prime} \mathrm{E}$ Long), N168/736997. Coll. by R. L. Kite and M. T. Te Punga.

\section{NZ-25. Christchurch}

Muddy peat in alluvial gravel, sand and silt, encountered in well $190 \mathrm{ft}$ below surface which is $45 \mathrm{ft}$ above sealevel, Corner of Blighs Road and Wairaki Rd., Christchurch (43 $30^{\prime}$ S Lat, $172^{\circ} 36^{\prime}$ E Long), S84/972594. Coll. by B. W. Collins N. Z. Geol. Surv., Christchurch.

\section{NZ-26. Christchurch}

Wood, $11 \mathrm{ft}$ below surface, in alluvium near base of Port Hills $\mathrm{fm}$, exposed in basement excavation at alt $20 \mathrm{ft}$ above sealevel, Cashmere Hospital, Christchurch (43 $34^{\prime}$ S Lat, $172^{\circ} 37^{\prime}$ E Long), S84/989514. Coll. by B. W. Collins.

\section{NZ-27. Christchurch}

Wood, $8 \mathrm{ft}$ below surface, in silty sand, exposed in excavation; $25 \mathrm{ft}$ above sealevel; St Asaph St., Christchurch ( $43^{\circ} 32^{\prime}$ S Lat, $172^{\circ} 38^{\prime}$ E Long), S84/ 995555. Coll. by B. W. Collins.

\section{NZ-28. Wairakei}

$5920 \pm 100$ 3970 B.C.

Wood from core, $200 \mathrm{ft}$ below surface, in pumiceous debris, bore 4 , Wairakei Geothermal Proj. (38 $38^{\prime} \mathrm{S}$ Lat, $176^{\circ} 05^{\prime} \mathrm{E}$ Long), N94/562453. Coll. by J. Healy. Comment: date gives rate of accumulation of tuffaceous and alluvial debris in the Wairakei Basin.

\section{NZ-29. North Auckland}

Kauri (Agathis australis) wood imbedded in indurated humus and iron pan, One Tree Point, Ruakaka (35 $40^{\prime}$ S Lat, $174^{\circ} 27^{\prime}$ E Long), N24/967859. Coll. by N. H. Taylor. Comment: pan horizon is almost certainly part of a podzol that existed when One Tree Point was more extensive than at present. Result is average of two determinations, 35,000 \pm 2000 and $34,600 \pm 1000$. 


\section{NZ-30. Hutt Valley}

NZ-31. Hutt Valley
$4470 \pm 100$

2520 в.c.

$4400 \pm 100$

2450 в.C.

Wood from two matai (Podocarpus spicatus) stumps in growth position at base of Melling Terrace, just $\mathrm{N}$ of Old Melling Bridge ( $41^{\circ} 12^{\prime} \mathrm{S}$ Lat, $174^{\circ}$ 55' E Long), N160/458324. Stumps were at present-day Hutt River level, which is at MHW, $17 \mathrm{ft}$ below terrace top and $50 \mathrm{yd}$ apart. Seeds, leaves, and pollen in peat at same horizon give some indication of climate warmer than present. Coll. by G. R. Stevens, N. Z. Geol. Surv.

\section{NZ-32. Victoria, Australia}

Modern

Wood from central, most-decomposed part of root of standing Eucalyptus oleosa, ca. $4 \mathrm{ft}$ in diam, $40 \mathrm{ft}$ high, of "bull-mallee" habit, on research farm of Victoria Dept. of Agriculture ( $35^{\circ} 10^{\prime} \mathrm{S}$ Lat, $142^{\circ} 00^{\prime} \mathrm{E}$ Long). Tree was suspected to be several thousand years old. Coll. by A. B. Costin, Soil Conserv. Authority, Victoria.

\section{NZ-33. Mount Gambier, Australia}

$4830 \pm 70$

2280 в.c.

Charcoal from A horizon of fossil soil immediately below ash of Mount Gambier volcano, 46 in. below surface $\left(37^{\circ} 49^{\prime} \mathrm{S}\right.$ Lat, $140^{\circ} 46^{\prime} \mathrm{E}$ Long). Soil profile above sample consists, from top downward, of gray-brown organic loam, 7 in. yellow-brown loam, 15 in. yellow-brown loam (weathered ash), 7 in., and dark, gray-speckled, stratified volcanic ash, 17 in. Subm. by E. D. Gill, Natl. Mus. of Victoria, Melbourne. Comment: sample dates the A horizon, indicates maximum age of volcanism, and gives upper limit of time needed to form the overlying loam.

\section{NZ-34. Lake Eyre, South Australia \\ $19,200 \pm 500$ \\ 17,250 в.c.}

Organic matter (leaves, insects, and microorganisms) found in sulfur deposit at Lake Eyre ( $28^{\circ} 58^{\prime} \mathrm{S}$ Lat, $137^{\circ} 41^{\prime} \mathrm{E}$ Long). Carbon content of sulfur sample, $0.3 \%$. Coll. by I. Kaplan, Fisheries Div. CSIRO, Sydney.

NZ-37. Ngaruawhaia

$1780 \pm 60$

\section{A.D. 170}

Charcoal lumps imbedded in thin layer of pumice conglomerate, $16 \mathrm{ft}$ below surface, within gritty quartzose pumiceous sands, in boring on regatta grounds, Ngaruawhaia, near junction of Waipa and Waikato Rivers $\left(37^{\circ} 40^{\prime}\right.$ S Lat, $175^{\circ} 09^{\prime}$ E Long), N56/659624. Coll. by J. C. Schofield, N. Z. Geol. Surv. Comment: date is that of Taupo lapilli member and probably also that of pumice flood in the Waikato River.

NZ-38. Waikato

$1800 \pm 70$

A.D. 150

Charcoal from white water-sorted pumice layer in quarry, Hinton Gully ( $37^{\circ} 49^{\prime}$ S Lat, $175^{\circ} 20^{\prime}$ E Long), N65/837449. Coll. by J. C. Schofield.

NZ-39. Hope River

$\mathbf{8 5 0} \pm \mathbf{5 0}$

A.D. 1100

Wood fragments from stems up to $5 \mathrm{in}$. diam, $\mathrm{S}$ bank Hope River, $0.5 \mathrm{mi}$ 
upstream from Lewis Pass highway bridge ( $42^{\circ} 36^{\prime} \mathrm{S} \mathrm{Lat}, 172^{\circ} 27^{\prime} \mathrm{E}$ Long), S53/848703. Coll. by M. Gage, Canterbury Univ., Christchurch. Comment: apparently dates a recent landslide.

NZ-40. Cox Creek

Wood, $130 \mathrm{ft}$ above sealevel, $25 \mathrm{ft}$ below top of marine gravel, in road cut just $\mathrm{N}$ of Cox Creek (42 ${ }^{\circ} 19^{\prime} \mathrm{S}$ Lat, $171^{\circ} 16^{\prime} \mathrm{E}$ Long), S44/792038. Coll. by R. P. Suggate.

\section{NZ-41. Johnsonville}

$20,900 \pm 300$ 18,950 в.с.

Wood from $\mathrm{W}$ side of Porirua-Johnsonville motorway cut, $0.25 \mathrm{mi} \mathrm{N}$ of Takapu Road (41 $11^{\circ}$ ' S Lat, 174 ${ }^{\circ} 50^{\prime}$ E Long), N160/384348. Wood and associated leaves and seeds suggest climate colder than the present one. Coll. by J. W. Brodie, D. R. McQueen, and W. P. Tolley.

\section{NZ-42. Johnsonville}

Wood, associated with sand and rounded gravel in road cut, 4 chains $\mathrm{S}$ of old bridge $\mathrm{N}$ of Johnsonville Railway Station $\left(41^{\circ} 13^{\prime} \mathrm{S}\right.$ Lat, $174^{\circ} 48^{\prime} \mathrm{E}$ Long), N160/364300. Associated seeds, leaves and pollen indicate temperate climate. Coll. by J. W. Brodie, D. R. McQueen, and W. P. Tolley.

\section{NZ-43. Hutt Valley}

Wood from peat bed between gravel, which overlies greywacke and underlies solifluction debris, in road cut, Foster Crescent, Belmont $\left(41^{\circ} 11^{\prime} \mathrm{S}\right.$ Lat, $174^{\circ} 55^{\prime} \mathrm{E}$ Long), N160/470340. Pollen from peat indicates a climate similar to the present one. Coll. by G. R. Stevens.

\section{NZ-44. Hutt Valley}

Wood, on Haywards-Pauatahanui Rd., opposite Haywards Substation housing settlement $\left(41^{\circ} 09^{\prime} \mathrm{S}\right.$ Lat, $174^{\circ} 59^{\prime} \mathrm{E}$ Long), N160/520390. Associated leaves, seeds, and pollen indicate a climate cooler than the present one. Coll. by J. W. Brodie, D. R. McQueen, and W. P. Tolley.

\section{NZ-45. Hauraki Plains}

$$
\begin{gathered}
2370 \pm 70 \\
420 \text { в.с. }
\end{gathered}
$$

Shells from pit in shell deposit, ca. $10 \mathrm{ft}$ above sealevel, Kopuarahi, $\left(37^{\circ}\right.$ 14' S Lat, $175^{\circ} 30^{\prime}$ E Long), N49/003140. Coll. by D. Kear and J. C. Schofield.

\section{NZ-46. South Canterbury}

$$
\begin{array}{r}
1450 \\
\text { A.D. } 500
\end{array}
$$

Podocarpus totara log lying on surface, probably having moved downslope, $3500 \mathrm{ft}$ above sealevel, $0.5 \mathrm{mi}$ NNE Trig B, Block IV, Burke S. D., Canterbury, on E face of Mount Dalgety $0.5 \mathrm{mi}$ S of MacKenzie Pass $\left(45^{\circ} 44^{\prime}\right.$ S Lat, $170^{\circ} 27^{\prime}$ E Long). Sample came from outside of log, which was charred, presumably by tussock fires; growth rate of tree was ca. 2.75 in. in $160 \mathrm{yr}$ Coll. by J. D. Raeside, N. Z. Soil Bur., DSIR. Comment: present vegetation is snow tussock and fescue tussock, greatly reduced by fire and erosion. No living totara has been observed on this range. 
Podocarpus totara log lying on surface, $2350 \mathrm{ft}$ above sealevel, $1.5 \mathrm{mi} \mathrm{SE}$ Trig P, Block IX, Nevis S. D., Otago, on L bank of Doolan's Creek ( $45^{\circ} 09^{\prime} \mathrm{S}$ Lat, $168^{\circ} 58^{\prime}$ E Long). Present vegetation is chiefly Festuca novae-zealandiae with scattered Danthonia flavescens and matagouri scrub; site is somewhat stony. Coll. by P. Wardle, Univ. of Otago. Comment: no living totara in Nevis catchment.

\section{NZ-48. Eastern Otago}

Dacrydium biforme log, $7 \mathrm{ft}$ long, diam 9 in., growth rate 3.25 in. for ca. 240 rings, $2300 \mathrm{ft}$ above sealevel, Maungatua Range, W edge of Taieri Plain ( $45^{\circ} 52^{\prime} \mathrm{S}$ Lat, $170^{\circ} 09^{\prime} \mathrm{E}$ Long), S163/847728. No living Dacrydium trees in the neighborhood. Coll. by A. R. Mark, Univ. of Otago.

\section{NZ-49. Egmont}

$8200 \pm 150$

6250 в.C.

Charred wood from stream bank, on branch of Waiwakaiho River, $100 \mathrm{ft}$ upstream from junction with main stream ( $39^{\circ} 16^{\prime} \mathrm{S}$ Lat, $174^{\circ} 05^{\prime} \mathrm{E}$ Long), N119/668673. Specimen comprised two fragments from dead trunk in growth position and washed clean; it marks a forest layer buried by a young lahar from Mount Egmont. Coll. by J. T. Salmon, Victoria Univ. College, Wellington.

\section{NZ-50. Blenheim}

\section{A.D. 1100}

$\mathbf{8 5 0} \pm \mathbf{5 0}$

Charcoal from deep oven, 17 to 30 in. below surface, in early Maori moahunter site, Wairau bar $\left(41^{\circ} 31^{\prime} \mathrm{S}\right.$ Lat, $174^{\circ} 04^{\prime} \mathrm{E}$ Long). Coll. by R. Duff, Canterbury Mus., Christchurch.

\section{Te Anau series}

Organic material from the higher of two shelters used during recent Maori occupation of the high valley of Te Anau (45 $17^{\circ} \mathrm{S}$ Lat, $167^{\circ} 40^{\prime} \mathrm{E}$ Long). Coll. by K. Miers, Wild Life Div., N. Z. Dept. of Int. Affairs.

\section{NZ-51. Tussock}

Tussock, presumably used as bedding.

\section{NZ-52. Totara}

$$
\mathbf{8 3 0} \pm \mathbf{5 0}
$$

Totara hark, presumably used as containers for preserved birds. Average of two runs, $820 \pm 60$ and $840 \pm 60$.

\section{NZ-53. Hina Hina}

$740 \pm 75$

\section{A.D. 1210}

Charcoal from lowest charcoal stratum, $4 \mathrm{ft}$ below surface, on clean sand and ash with few shells, Hina Hina moa-hunter site, $0.5 \mathrm{mi}$ from Pounawea moa-hunter site, Papatowai (46 $29^{\prime} \mathrm{S}$ Lat, $169^{\circ} 42^{\prime} \mathrm{E}$ Long), S184/485966. Coll. by L. Lockerbie, Otago Mus. School Serv., Dunedin. Comment: tree roots extend to sampled level. Although the area receives relatively high rainfall, the sand is usually dry. Sampled stratum lies several feet above high-tide level. 


\section{Pounawea series, Otago}

Samples from Pounawea moa-hunter site at junction of Catlins and Owaka Rivers, Papatowai $\left(46^{\circ} 28^{\prime} \mathrm{S}\right.$ Lat, $169^{\circ} 42^{\prime} \mathrm{E}$ Long), S184/486975. Site is in a high-rainfall district, low-lying but well drained. Stratification below present forest (chiefly Podocarpus totara) is: (a) top stratum, chiefly of loose shells with fish, seal, whale, and bird bones; artifacts of bone and stone throughout (b) intermediate stratum of fine gray ash and sand containing charcoal, bones (including moa), moa eggshell, a few seashells, and many artifacts (c) bottom stratum of black, greasy ash and sand containing charcoal and bird, seal, whale, fish, and moa bones; shells not numerous; flake knives and other artifacts present. Significant dietary changes are evident; date of abandonment of site is apparently confirmed by ages of totara trees growing on the deposit. Coll. by L. Lockerbie.

\section{NZ-54. Pounawea, upper shell}

Shell from 2 in. below surface in top stratum.

\section{NZ-55. Pounawea, intermediate charcoal}

Charcoal from deposit in intermediate stratum.

NZ-56. Pounawea, seal carbonate fraction

\section{NZ-56. Pounawea, seal organic fraction}

A.D. 1560

$$
390 \pm 60
$$

$$
520 \pm 55
$$

A.D. 1430

A.D. 1430

$$
\mathbf{5 5 0} \pm \mathbf{5 5}
$$

A.D. 1400

Carbonate and organic fractions of seal bones from intermediate stratum.

\section{NZ-57. Pounawea, lower shell}

Shell from lowest shell deposit.

NZ-58. Pounawea, lower charcoal
A.D. 1350

$$
600 \pm 60
$$

A.D. 1140

Charcoal from bottom of deposit, resting on unconsolidated river silt.

\section{Hawksburn Valley series, central Otago}

Samples from moa-hunter site on terrace in narrow part of Hawksburn Valley, $2050 \mathrm{ft}$ above sealevel, Carrick Mountains $\left(45^{\circ} 13^{\prime} \mathrm{S} \mathrm{Lat}, 169^{\circ} 10^{\prime} \mathrm{E}\right.$ Long). Steep slopes, fringing cliffs, and swamps provide a natural trap for moas; bones and wood would be expected to remain on surface for a long time, as district has little rainfall (10 to $12 \mathrm{in.}$.), low tussock vegetation, slow soil formation, and extreme winter frosts. Climatic changes are indicated by occurrence of totara logs at alt $3000 \mathrm{ft}$, which is above present limit of growth. Coll. by L. Lockerbie.

\section{NZ-59. Hawksburn, burnt bone carbonate A.D. 1540 \\ NZ-59. Hawksburn, burnt bone organic matter \\ $410 \pm 55$$$
400 \pm 55
$$ \\ A.D. 1550}

Burnt moa bone from oven containing other moa (unburnt) bones, a 
canid jaw, and stone artifacts, surrounded by dry loess, surface cover tussock and scabweed. NZ-60 (unburnt) was mixed with this sample before processing.

NZ-60. Hawksburn, Euryapteryx bone

$450 \pm 60$

A.D. 1500

Unburnt bone, a femur of Euryapteryx, 6 to $7 \mathrm{in}$. below surface, in very dry, dusty soil, associated with jasperoid and quartzite artifacts.

NZ-61. Hawksburn, wood

$\mathbf{5 9 0} \pm \mathbf{5 0}$

A.D. 1360

Charred, well-preserved wood from bottom of occupation deposit, 26 in. below surface, on blue clay, associated with artifacts and moa-bone fragments.

\section{NZ-62. Hawksburn, charcoal}

A.D.1350

$600 \pm 60$

Charcoal from upper 6 in. of occupation layer.

\section{Egmont series}

Charcoal from a Maori oven, overlain by volcanic ash, 15 in. thick, part of the Burrell ash shower, Egmont $\left(39^{\circ} 18^{\prime} \mathrm{S}\right.$ Lat, $174^{\circ} 07^{\prime} \mathrm{E}$ Long), N119/ 701617. Samples antedate the ash, and, being firewood, may also antedate the oven. Coll. by H. S. Gibbs, N. Z. Soil Bur., DSIR.

\section{NZ-63. Egmont}

Charcoal from large piece in centre of oven.

\section{NZ-64. Egmont}

Small pieces of charred wood from various parts of oven.

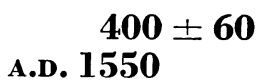

$360 \pm 60$

\section{NZ-65. Te Horo}

Wood from lignitic layer, 18 to 24 in. thick, overlying marine sediments with sponge spicules, overlain by sandstone, $10 \mathrm{ft}$ thick, and then by angular solifluxion debris, 30 in. thick, exposed in ancient seacliff, $2 \mathrm{mi} 30$ chains at $215^{\circ}$ from Te Horo Railway Station ( $40^{\circ} 50^{\prime} \mathrm{S}$ Lat, $175^{\circ} 06^{\prime}$ E Long), N157/ 625772. Coll. by M. T. Te Punga.

\section{Lake Merindee series, South Australia}

Unionid shells from archaeologic site, Lake Merindee $\left(32^{\circ} 20^{\prime} \mathrm{S}\right.$ Lat, $142^{\circ}$ $25^{\prime}$ E Long). Dates are calculated from $\mathrm{C}^{14}$ content of modern Unio shells from Tartanga lagoon on Murray River, which was $1.70 \pm 0.4 \%$ above NZ modern wood standard. Coll. by L. F. Marcus, South Australia Mus., Adelaide.

\section{NZ-66. Lake Merindee, Layer B, Area I $\quad 6570 \pm 100$}

Shells, collected in situ and broken from matrix, accompanied by extinct genera of Late Pleistocene or recent mammals and by artifacts of Tartangan culture. 


\section{NZ-67. Lake Merindee, Layer B, Area II}

Shells from Layer B, on surface in an area 15 by $5 \mathrm{yd}$; some were collected in situ but all were friable and easily separated from matrix. Four Rat Kangaroo mandibles and a maxillary fragment were found in association on surface.

NZ-68. Lake Merindee, Layer O, Area IV

Modern

Shells, mainly broken, lying on surface, on Layer O, wind-blown, loose, coarse sand, ca. 9 in. deep. Fauna of Layer $O$ comprised only living species of mammals; associated artifacts were of Mudukian culture. Comment: a few artifacts found on the site suggest the Kartan culture, which is probably Late Pleistocene, at least at its beginning. The Tartangan date quoted above was for a middle horizon of the culture, and agrees closely with NZ-66. The much younger, microlithic Mudukian culture has been shown elsewhere to postdate the Pirrian culture, a mid-point of which was dated at Devon Downs at 4250 $\pm 180\left(32^{\circ} 20^{\prime} \mathrm{S}\right.$ Lat, $142^{\circ} 25^{\prime}$ E Long.)

\section{NZ-69. Cape Martin, South Australia}

$$
\begin{aligned}
& 8800 \pm 120 \\
& 6850 \text { B.C. }
\end{aligned}
$$

Charcoal, separated by washing from a hearth at same horizon as Tartan. gan artifacts, in Layer B, a red, earthy deposit containing dominantly estuarine shells, overlain by white dune sand on which there is a site of Murundian culture containing reef shells similar to those now living at Cape Martin $\left(37^{\circ}\right.$ $29^{\prime}$ S Lat, $140^{\circ} 01^{\prime}$ E Long). Coll. by N. B. Tindale, South Australia Mus., Adelaide.

\section{Eromanga series, Queensland}

Calcium carbonate deposited from artesian water, stored for several years before measurement. Coll. by H. C. Webster, Univ. of Queensland.

NZ-70. Eromanga

Carbonate from bore pipe, diam 6 in., which originally supplied township of Eromanga $\left(26^{\circ} 42^{\prime} \mathrm{S}\right.$ Lat, $143^{\circ} 48^{\prime} \mathrm{E}$ Long).

\section{Harper River series}

Wood from a probable major slump at junction of $\mathrm{E}$ and $\mathrm{W}$ Branches of Harper River ( $43^{\circ} 11^{\prime} \mathrm{S}$ Lat, $171^{\circ} 33^{\prime} \mathrm{E}$ Long), S66/554555. The area is close to a major fault (Harper Fault), involving late Tertiary beds (Suggate, 1958a). Coll. by R. P. Suggate and D. Wilson, N. Z. Geol. Surv., DSIR.

\section{NZ-72. Harper River, $50 \mathrm{ft}$ above river level $\quad 4620 \pm 80$ \\ $4550 \pm 80$ \\ 2600 B.C.}

NZ-74. Johnsonville

Wood, part of a temperate flora, at road level, Porirua-Johnsonville motorway, 1st cut on E side, S of Porirua Railway Station, $75 \mathrm{ft}$ above sealevel $\left(41^{\circ}\right.$ 09' S Lat, $174^{\circ} 51^{\prime}$ E Long), N160/397395. Coll. hy D. R. McQueen. 


\section{NZ-75. Ricearton}

$$
3570 \pm 70
$$

\section{0 в.C.}

Wood from peat bed in silt underlying Canterbury Plains surface, Mandeville Road, depth $12 \mathrm{ft}$, Bore No. 2 ( $43^{\circ} 32^{\prime} \mathrm{S}$ Lat, $172^{\circ} 36^{\prime}$ E Long), S84/ 979558. Coll. by P. J. Alley, Canterbury Univ. College, Christchurch.

\section{Jerf Ajla series, Syria}

Finely divided charcoal, mixed with dirt, from Jerf Ajla, a cave in the Syrian Desert ( $34^{\circ} 38^{\prime} \mathrm{N}$ Lat, $37^{\circ} 08^{\prime} \mathrm{E}$ Long). The two samples (A and C) were expected to be of the same age, older than $30,000 \mathrm{yr}$, and to settle the question of late persistence of Levalloiso-Mousterian culture in the district. Carbon content after treatment with $\mathrm{HCl}$, ca. $25 \%$. Coll. by C. S. Coon, Univ. Mus., Univ. of Pennsylvania, Philadelphia, Penn., U. S. A.

\section{NZ-76. Sample A-8, charcoal}

\section{NZ-77. Sample C-8, charcoal}

\section{NZ-78. Sample C-8, carbon dioxide}

$\mathrm{CO}_{2}$ evolved from sample NZ-77 during $\mathrm{HCl}$ treatment.
$43,000 \pm 2000$ 41,050 в.с.

$$
\begin{aligned}
& 18,000 \pm 200 \\
& 16,050 \text { B.C. }
\end{aligned}
$$$$
19,800 \pm 300
$$$$
17,850 \text { в.с. }
$$

NZ-79. Auckland

Carbonized wood from well-defined seam, 1 to 3 in. thick, ca. $25 \mathrm{ft}$ below surface, $10 \mathrm{ft}$ below upper level of Waitemata formation, Civic Sq., Auckland ( $36^{\circ} 5 l^{\prime}$ S Lat, 174 $46^{\prime}$ E Long), N42/283605. Coll. by Ministry of Works, Auckland.

NZ-80. Awahuri

Wood, depth $233 \mathrm{ft}$ in Dairy Factory Well, Awahuri $\left(40^{\circ} 18^{\prime} \mathrm{S}\right.$ Lat, $175^{\circ}$ 32' E Long), N149/033427. Coll. by M. T. Te Punga.

\section{NZ-81. Foxton}

$$
9900 \pm 150
$$

\section{0 в.с.}

Wood, depth $155 \mathrm{ft}$ (150 ft below sealevel) in well near water tower. Foxton $\left(40^{\circ} 29^{\prime} \mathrm{S}\right.$ Lat, $175^{\circ} 17^{\prime} \mathrm{E}$ Long), N148/796206. Coll. by M. T. Te Punga.

\section{NZ-82. Waiora Valley, Wairakei}

$$
2000 \pm 100
$$

50 в.c.

Wood from small $\log$ in cave at $\mathrm{W}$ end of Alum Lake at head of Waiora Valley ( $38^{\circ} 37^{\prime} \mathrm{S}$ Lat, $176^{\circ} 04^{\prime} \mathrm{E}$ Long), N94/533460. Dates a volcanic eruption in Waiora Valley. Coll. by C. J. Banwell, Dominion Physical Lab., DSIR.

\section{NZ-84. Christchurch}

$$
\begin{array}{r}
1550 \pm 80 \\
\text { A.D. } 400
\end{array}
$$

Wood, depth 11 to $15 \mathrm{ft}$ in boiler foundation excavation, Public Hospital, Christchurch $\left(43^{\circ} 32^{\prime} \mathrm{S}\right.$ Lat, $172^{\circ} 38^{\prime}$ E Long), S84/995558. See Suggate, 1958b for details. Coll. by B. W. Collins. 
NZ-85. Terrace-Bowen Street. Wellington

Wood, depth $65 \mathrm{ft}$ in drill hole for building foundation, in mid-Pleistocene Emerald Terrace, Terrace-Bowen Street, Wellington $\left(41^{\circ} 17^{\prime} \mathrm{S}\right.$ Lat, $174^{\circ} 47^{\prime}$ E Long), N164/336229. Coll. by H. W. Wellman, Victoria Univ., Wellington.

\section{NZ-86. Bowenvale Road, Christchurch}

Wood, $8 \mathrm{ft}$ below road level, $\mathrm{S}$ abutment of Heathcote Bridge, Bowenvale Rd., Christchurch (43 $34^{\prime}$ S Lat, $172^{\circ} 39^{\prime}$ E Long), S84/009519. Coll. by B. W. Collins.

\section{NZ-87. Herbert Street, Wellington}

Wood, depth $35 \mathrm{ft}$ in drill hole for building foundation, in Upper Pleistocene Whiteman Terrace, Herbert Street, $20 \mathrm{ft}$ from W corner of Dixon St., Wellington $\left(41^{\circ} 18^{\prime} \mathrm{S}\right.$ Lat, $174^{\circ} 47^{\prime}$ E Long), N164/336214. Coll. by R. W. Willett and G. J. Lensen, N. Z. Geol. Surv., DSIR.

\section{NZ-88. Lake Hawea}

$15,100 \pm 200$

Peat from bottom of peat bed deposited against old slump at outlet of glacial Lake Hawea (44 $37^{\prime}$ S Lat, $169^{\circ} 15^{\prime}$ E Long). Coll. by I. C. McKellar, N. Z. Geol. Surv., DSIR. Comment: dates early part of retreat of N. Z. glaciers at end of last glaciation (McKellar, 1960).

\section{NZ-89. Silver Peaks, Dunedin}

\section{A.D. 1300}

$650 \pm 60$

Wood (Podocarpus totara), $2300 \mathrm{ft}$ above sealevel, on hillside now carrying snow tussock and silver beech, Silver Peaks Dist., Dunedin $\left(45^{\circ} 44^{\prime} \mathrm{S}\right.$ Lat, $170^{\circ} 27^{\prime} \mathrm{E}$ Long), S164/100880. Dates a period of climate moister than the present one. Coll. by P. Wardle, Univ. of Otago.

\section{NZ-90. The Gap, Silver Peaks, Dunedin}

Wood (Podocarpus totara), $1850 \mathrm{ft}$ above sealevel, on hillside now carrying snowgrass, The Gap, Silver Peaks-Dist., Dunedin $\left(45^{\circ} 42^{\prime} \mathrm{S}\right.$ Lat, $170^{\circ} 28^{\prime}$ E Long), S155/120930. Some silver beech in vicinity, but no other trees; totara dates a period of climate moister than the present one. Coll. by G. T. S. Bayliss, Univ. of Otago.

\section{NZ-91. Waverly}

$$
\begin{array}{r}
1020 \\
\text { A.D. } 930
\end{array}
$$

Wood from upright tree trunk, part of a drowned forest, in river bed at mouth of Waitotara River, Waverley ( $39^{\circ} 51^{\prime}$ S Lat, $174^{\circ} 41^{\prime}$ E Long), N137/ 239967. Coll. by C. A. Fleming. Comment: date, and stratigraphic situation, suggest subsidence of ca. $10 \mathrm{ft}$ in the last $1000 \mathrm{yr}$.

\section{NZ-92. Wanganui}

Wood from youngest band in lignite bed, in Kaiwhara alluvium, the youngest member of the Rapanui formation, Landguard Bluff, Wanganui $\left(39^{\circ}\right.$ $57^{\prime} \mathrm{S}$ Lat, $175^{\circ} 01^{\prime} \mathrm{E}$ Long), N138/553837. Alluvium was deposited during a period of slight climatic amelioration after a period of river entrenchment through the marine Waipuna Delta conglomerate. Coll. by C. A. Fleming. 


\section{Canterbury Plains series}

Wood and peat in alluvium, sampled to date the Otarama surface and the younger Kowhai River Terrace that overlies it, Canterbury Plains. Coll. by M. Gage, B. W. Collins, R. P. Suggate, and R. W. Willett.

\section{NZ-93. Rubicon River}

Peat, overlain by Kowhai River Terrace, S bank Rubicon River $\left(43^{\circ} 19^{\prime}\right.$ S Lat, $171^{\circ} 53^{\prime}$ E Long), S74/338832.

\section{NZ-94. Joyce Stream}

Wood and peat, $200 \mathrm{ft}$ below Otarama surface, $\mathrm{N}$ bank Joyce Stream ( $43^{\circ} 17^{\prime}$ S Lat, $171^{\circ} 57^{\prime}$ E Long), S74/394868.

NZ-95. Joyce Stream, wood

Wood, ca. $200 \mathrm{ft}$ below Otarama surface, $\mathrm{N}$ bank Joyce Stream $0.25 \mathrm{mi}$ upstream from ford on disused Otarama Rd. $\left(43^{\circ} 17^{\prime} \mathrm{S}\right.$ Lat, $171^{\circ} 57^{\prime} \mathrm{W}$ Long), S74/394868.

NZ-96. Joyce Stream, peat

Peat, same locality and stratgiraphic position as NZ-95.

\section{NZ-97. Rubicon River, wood}

Wood, $10 \mathrm{ft}$ below surface of main Kowhai River Terrace, overlying Otarama surface, $\mathrm{S}$ bank Rubicon River, $0.25 \mathrm{mi}$ downstream from homestead

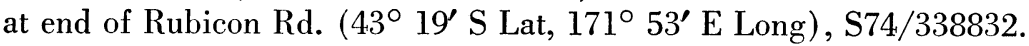

\section{NZ-98. Rubicon River, peat}

Peat, same locality and stratigraphic position as NZ-97.

\section{NZ-99. Blighs Road, Christchurch}

Peat, depth $150 \mathrm{ft}$ in well, Blighs Rd., Christchurch $\left(43^{\circ} 30^{\prime} \mathrm{S}\right.$ Lat, $172^{\circ}$ $36^{\prime}$ E Long), S84/972504. Sampled to determine position of hypothetical unconformity between postglacial alluvial and marine sediments (Suggate, 1958b). Coll. by R. P. Suggate.

\section{NZ-100. Lake Waikaremoana}

$$
\begin{gathered}
2200 \pm 60 \\
250 \text { в.C. }
\end{gathered}
$$

Wood from standing tree, drowned by rise of lake, exposed by lowering of lake level by ca. $50 \mathrm{ft}$, $\mathrm{N}$ shore of Lake Waikaremoana, $2.5 \mathrm{mi}$ WNW of Lake House ( $38^{\circ} 45^{\prime} \mathrm{S}$ Lat, $177^{\circ} 07^{\prime}$ E Long), N105/532295. Coll. by H. W. Wellman.

\section{NZ-101. Lake Waikaremoana}

$$
2190 \pm 60
$$

Wood from another tree at same locality as NZ-100.

\section{Takapu Road series, Johnsonville}

Wood from clay fill in V-shaped gully cut in greywacke, Takapu Rd., Johnsonville $\left(41^{\circ} 11^{\prime} \mathrm{S}\right.$ Lat, $174^{\circ} 50^{\prime}$ E Long), N160/385348. Sampled to 
show rate and continuity of deposition of fill, in connection with study of former morphology and drainage in the district. Coll. by J. W. Brodie.

\section{NZ-102. Takapu Road, uppermost horizon}

Sample R160/1.

NZ-103. Takapu Road, uppermost horizon

Duplicate of R160/1, NZ-102.

NZ-104. Takapu Road, 2.5 ft

Wood from $2.5 \mathrm{ft}$ below R160/1.

NZ-105. Takapu Road, $7 \mathrm{ft}$

Wood from $7 \mathrm{ft}$ below R160/1.

NZ-106. Takapu Road, base
$21,500 \pm 350$

19,550 в.с.

$21,300 \pm 350$

19,350 в.с.

$22,300 \pm 350$ 20,350 в.c.

$22,800 \pm 350$ 20,850 в.c.

$37,500 \pm 1600$ $\mathbf{3 5 , 5 5 0}$ в.с.

From angular conglomerate at base of fill, 14 ft below R160/1.

\section{NZ-107. Kahao Creek}

$1650 \pm 60$

A.D. 300

Wood from layer of logs, $3 \mathrm{ft}$ below surface of aggradation terrace at present stream level, Kahao Creek ( $41^{\circ} 05^{\prime} \mathrm{S}$ Lat, $174^{\circ} 54^{\prime}$ E Long), N160/ 446468. Locality described by Brodie, 1957. Coll. by J. W. Brodie.

NZ-108. Porirua

$>41,000$

Peat from clay layer, overlain by angular fragments, overlying gravel, another bed of clay with plant remains, another gravel layer, and the wood dated as NZ-74, on Porirua-Johnsonville Rd., same locality as NZ-74 ( $>45,000$, this date list). Coll. by J. W. Brodie.

\section{Karori series}

Organic material, part of a suspected fossil soil, $5 \mathrm{ft}$ below surface, overlain by weathered clay, Karori $\left(41^{\circ} 17^{\prime} \mathrm{S}\right.$ Lat, $174^{\circ} 44^{\prime} \mathrm{E}$ Long), N164/ 305224. Coll. by J. W. Brodie.

NZ-109. Karori, washed sample

NZ-110. Karori, sorted sample

Comment: material is probably of interstadial age.

\section{NZ-111. Wainuiomata, Wellington}

$$
\begin{aligned}
& \mathbf{2 4 , 0 0 0} \pm 500 \\
& \mathbf{2 2 , 0 5 0} \text { в.с. } \\
& \mathbf{2 7 , 0 0 0} \pm \mathbf{6 0 0} \\
& \mathbf{2 5 , 0 5 0} \text { в.с. }
\end{aligned}
$$

$7230 \pm 100$ $\mathbf{5 2 8 0}$ в.c.

Wood with bark, size $8 \times 4 \times 3.5 \mathrm{in}$. at top of peat, overlain by $5 \mathrm{ft}$ of silt. In W bank Black Creek opposite Kent St., Wainuiomata $\left(41^{\circ} 15^{\prime} \mathrm{S}\right.$ Lat, $174^{\circ} 44^{\prime}$ E Long), N164/491272. Coll. by M. T. Te Punga. Comment: dates close of peat formation. 


\section{NZ-112. Wainuiomata, Wellington}

Wood from branch in peat $5 \mathrm{ft}$ thick, $4 \mathrm{in}$. above bedrock, $400 \mathrm{yd} \mathrm{W}$ of Fitzherbert Rd. junction, beside Wainuiomata Rd. $\left(41^{\circ} 16^{\prime} \mathrm{S}\right.$ Lat, $174^{\circ} 56^{\prime}$ E Long). Coll. by M. T. Te Punga. Comment: date is early in period of peat formation and shortly after close of last local episode of "solifluction."

\section{NZ-113. Hawke Bay}

$$
2030 \pm 100
$$

80 в.c.

Carbonized wood: rolled fragments in flat pebbles of pumice from a depth of 30 fathoms in Hawke Bay ( $39^{\circ} 16^{\prime} \mathrm{S}$ Lat, $177^{\circ} 22^{\prime} \mathrm{E}$ Long). Coll. by H. M. Pantin, N. Z. Oceanog. Inst. Comment: date and lithology suggest this charcoal and pumice may have been deposited by a Taupo-Pumice flood. That the date is greater than that of NZ-1, 3, 4 etc. could be accounted for by derivation from center of a large tree.

\section{NZ-114. Muriwai Beach, Auckland}

$$
\begin{array}{r}
1030 \\
\text { A.D. } 920
\end{array}
$$

Shells of Amphidesma Subtriangulata from sub-fossil deposit behind foredunes, $10 \mathrm{mi} \mathrm{N}$ of beach road $\left(36^{\circ} 39^{\prime} \mathrm{S}\right.$ Lat, $174^{\circ} 19^{\prime} \mathrm{E}$ Long). Coll. by R. M. Cassie, N. Z. Oceanog. Inst. Comment: these shells are larger than contemporary shells and possibly belong to an extinct race.

\section{NZ-115. Hawera, Taranaki}

Wood in peat layer at depth of $35 \mathrm{ft}$ in a well. Peat overlies Upper Pleistocene lahars. Paora Rd., Hawera $\left(39^{\circ} 34^{\prime}\right.$ S Lat, 174 ${ }^{\circ} 14^{\prime}$ E Long), N129/ 813299. Coll. by P. G. Bamford. Ohawe Beach, Hawera. Comment: peat was formed during the first interstadial of the last glaciation.

\section{NZ-116. Greymouth \\ $22,300 \pm 350$ \\ 20,350 в.c.}

Wood from lacustrine sand and silt interbedded with gravel. Side of main Greymouth Reefton Rd., 2 chains toward Greymouth from Kamaka Sta. $\left(42^{\circ}\right.$ $26^{\prime}$ S Lat, $171^{\circ} 23^{\prime}$ E Long), S44/900909. Coll. by E. T. Annear. N. Z. Geol. Surv., Greymouth. Comment: sand and silt deposited during $\mathrm{K}_{2}$ advance within Kumara Glacial substage.

\section{Christchurch series}

Shell and wood at various places and positions near Christchurch taken to date postglacial rise of sealevel.

\section{NZ-117. Kaiapoi, Christehurch}

$6800 \pm 90$

4850 в.c.

Shell from $15 \mathrm{ft}$ below MSL in Moore's Gravel Pit, Kaiapoi $\left(43^{\circ} 22^{\prime} \mathrm{S}\right.$ Lat, $172^{\circ} 40^{\prime}$ E Long), S76/033773. Coll. by B. W. Collins. (Suggate, 1958b).

\section{NZ-118. Lake Ellesmere}

$9400 \pm 120$

7450 в.c.

Wood at depth $71 \mathrm{ft}$ below MSL in bore. Dept. of Agriculture plot, Lake Ellesmere $\left(43^{\circ} 44^{\prime} \mathrm{S}\right.$ Lat, $172^{\circ} 31^{\prime} \mathrm{E}$ Long), S83/891322. Coll. by R. P. Suggate. (Suggate, 1958b.) 


\section{NZ-119. Christchurch City}

Wood at depth $57.5 \mathrm{ft}$ below MSL in bore, corner Madras and Chester Sts., Christchurch City ( $43^{\circ} 32^{\prime}$ S Lat, $172^{\circ} 39^{\prime}$ E Long). Coll. by R. P. Suggate. (Suggate, 1958b.)

NZ-120. Christchurch City

$6200 \pm 120$

\section{NZ-121.}

5150 в.c.

NZ-120. shell from $11 \mathrm{ft}$ below MSL)

NZ-121. peat from $105 \mathrm{ft}$ below MSL)

In bore at Clarence Rd. Christchurch City $\left(43^{\circ} 32^{\prime} \mathrm{S}\right.$ Lat, $172^{\circ} 36^{\prime} \mathrm{E}$ Long), S84/974563. Coll. by L. E. Oborn, N. Z. Geol. Surv. (Suggate, 1958b.)

\section{NZ-122. Christehurch City}

$\mathbf{3 8 1 0} \pm \mathbf{7 0}$

1860 в.c.

Wood from $3 \mathrm{ft}$ below MSL in excavation, corner Woodham and Worcester Sts., Christchurch City $\left(43^{\circ} 32^{\prime}\right.$ S Lat, $172^{\circ} 41^{\prime}$ E Long), S84/ 040567. Coll. by Mr. Samson, Drainage Board, Christchurch. (Suggate, 1958b.)

\section{NZ-123. Southland}

Wood at base of $5 \mathrm{ft}$ of peat in bog, between Tanner and McKenzie Rds., Hokonui, Southland $\left(46^{\circ} 07^{\prime} \mathrm{S}\right.$ Lat, $168^{\circ} 27^{\prime}$ E Long), S169/425376. Coll. by I. C. McKellar.

\section{NZ-124. Rapahoe Beach, Westland}

$6500 \pm 100$ 4550 в.c.

Wood, perhaps root, $20 \mathrm{ft}$ below top of gravel terrace and $100 \mathrm{ft}$ above sealevel. Cliff overlooking Rapahoe Beach $\left(42^{\circ} 23^{\prime} \mathrm{S}\right.$ Lat, $171^{\circ} 13^{\prime} \mathrm{E}$ Long), S44/754967. Coll. by F. E. Bowen, N. Z. Geol. Surv.

\section{Mohaka River series}

NZ-125. Mohaka River

Wood from basal $2 \mathrm{ft}$ of gravels laid down during early part of last major aggradation in Mohaka Valley at coast, S side $\left(39^{\circ} 08^{\prime} \mathrm{S}\right.$ Lat, $177^{\circ} 10^{\prime} \mathrm{E}$ Long), N115/577824. Coll. by T. L. Grant-Taylor. Comment: trees probably killed during onset of cold of last glaciation, determination places a younger limit on beginning of last glaciation.

\section{NZ-126. Mohaka River}

Wood from $15 \mathrm{ft}$ thick clayey horizon in Mohaka aggradation terrace. Clay laid down during short interval of partial downcutting following deposition of fill of first terrace of last major fluvial aggradation. Mohaka Valley 1.5 mi from coast in gorge formed by small stream $\left(39^{\circ} 07^{\prime} \mathrm{S}\right.$ Lat, $177^{\circ} 10^{\prime} \mathrm{E}$ Long), N115/569835. Coll. by T. L. Grant-Taylor. Comment: because downcutting probably occurred during the first interstadial of the last glaciation, determination places a younger limit on the age of this interstadial. 
NZ-127. Hastings

$6620 \pm 100 \mathrm{ft}$

4670 в.с.

Shells from shell bed $60 \mathrm{ft}$ below MSL in Bore at N. Z. Aerial Mapping Ltd, Hastings $\left(39^{\circ} 39^{\prime} \mathrm{S}\right.$ Lat, $176^{\circ} 51^{\prime}$ E Long), N134/263213. Coll. by T. L. Grant-Taylor. Comment: shells show intertidal estuarine ecology, and probably were deposited on a beach. Although the area was affected by earth movements in 1929 , depth probably is accurate to ca. $10 \mathrm{ft}$, and date is that of a sealevel ca. $-60 \mathrm{ft}$.

NZ-128. Hawera

$$
1450 \pm 60
$$

A.D. 500

Wood from peat layer immediately above top lahar at end of Fairfield Rd., Hawera ( $39^{\circ} 37^{\prime} \mathrm{S}$ Lat, $174^{\circ} 17^{\prime} \mathrm{E}$ Long), N129/852240. Coll. by G. J. Lensen. Comment: a widespread ancient peat overlies the lahars in this area. This sample was separated from underlying lahars by a thin sand: peat probably from interdune swamp. See NZ-115.

\section{NZ-129. Mt. Maunganui}

$620 \pm 80$

Shells from shell bed $10 \mathrm{ft}$ above sealevel on SW coast of Mt. Maunganui ( $37^{\circ} 38^{\prime}$ S Lat, $176^{\circ} 10^{\prime}$ E Long), N58/645655. Coll. by D. Kear, N. Z. Geol. Surv., Auckland.

NZ-130. Weheka, Westland

Modern $<\mathbf{1 0 0}$

NZ-131

Modern $<\mathbf{1 0 0}$

Wood from gravel of low outwash terrace at depth $6 \mathrm{ft}$. R bank Bullock Creek, 50 yds above road bridge, ca. $5 \mathrm{mi} \mathrm{S}$ of Weheka (Fox Glacier), Westland $\left(43^{\circ} 35^{\prime} \mathrm{S}\right.$ Lat, $169^{\circ} 37^{\prime}$ E Long), S78/330490. Coll. by N. E. Odell. Comment: two determinations on separate pieces of wood.

\section{NZ-132. Bruce Bay, South Westland}

$3320 \pm 120$

1370 в.C.

Wood $3 \mathrm{ft}$ below surface in sandy marine terrace $7 \mathrm{ft}$ above sealevel. At Bruce Bay $1 \mathrm{mi} \mathrm{N}$ of township at junction of New and Old Shore Rds. $\left(43^{\circ} 35^{\prime}\right.$ S Lat, $169^{\circ} 37^{\prime}$ E Long), S75/330490. Coll. by N. E. Odell. Comment: dates uplift of terrace.

\section{NZ-133. Parawhakatau, Kaikoura Coast}

$$
\text { A.D. } 1630 \pm 60
$$

Wood from wallpost taken from House Pit $\mathrm{C}$ at Parawhakatau $1 \mathrm{mi} \mathrm{N}$ of Claveley, Kaikoura Coast $\left(42^{\circ} 33^{\prime}\right.$ S Lat, $173^{\circ} 30^{\prime}$ E Long). Coll. by R. E. Bell, Univ. of Oklahoma. Comment: legend suggests that the pa was occupied for ordy $20 \mathrm{yrs}$.

\section{NZ-134. Papatowai, Otago \\ A.D. 1185 \\ $765 \pm 30$}

\section{NZ-135. Duplicate sample}

Charcoal from lowest levels of occupation debris of a moa-hunter site at mouth of Tahakopa River, Papatowai, Otago $\left(46^{\circ} 34^{\prime} \mathrm{S}\right.$ Lat, $169^{\circ} 27^{\prime} \mathrm{E}$ Long). Coll by R. E. Bell. 
Tahakopa River Mouth series

Samples from two occupation levels, in a trench excavation with bones of Dinornis maximus and Eurapteryx gravis in an association that implies a later date for their extinction than was previously expected. Tahakopa River Mouth, Otago ( $46^{\circ} 34^{\prime} \mathrm{S}$ Lat, $169^{\circ} 27^{\prime}$ E Long). NZ-136 was from basal deposit; the others are from younger occupation, midway up cliff. Coll. by L. Lockerbie.

NZ-136. Base of trench

$$
\mathbf{6 3 0} \pm \mathbf{5 0}
$$

$$
\text { A.D. } 1320
$$

Charcoal associated with moa and seal bones from base of trench.

\section{NZ-137. Eurapteryx bone}

$$
460 \pm 50
$$

Eurapteryx gravis bones resting on layer of charcoal, ash and sand with bones and artifacts.

\section{NZ-138. Dinornis bone}

A.D. 1490

Dinornis maximus bone with seal bones and shell.

\section{NZ-139. Eurapteryx bone}

Eurapteryx gravis bones with moa and seal bones.

NZ-140. Moa bone

Species not determined.

$$
460 \pm 80
$$$$
310 \pm 60
$$

$$
390 \pm 80
$$

\section{False Island series}

Various samples from two ovens and associated refuse deposit. Deposit postdates use of moa as food at False Island, Otago $\left(46^{\circ} 29^{\prime} \mathrm{S}\right.$ Lat, $169^{\circ} 45^{\prime}$ E Long). Coll. by L. Lockerbie.

\section{NZ-141. Shell}

Amphidesma australe shells.

\section{NZ-142. Fishbones}

\section{NZ-143. Charcoal from oven}

\section{NZ-144. Charcoal not from oven}

\section{NZ-145. Charcoal from oven}

\section{NZ-146. Tautuku, Otago}

470
A.D. 1480

A.D. 1660

$$
290 \pm 80
$$

A.D. 1630

$$
\mathbf{3 2 0} \pm \mathbf{5 0}
$$

A.D. 1605

$$
\mathbf{3 4 5} \pm \mathbf{5 0}
$$

$\mathbf{2 1 5} \pm \mathbf{5 0}$

A.D. 1735

A.D. 1670

Bones of Dinornis torosus, an intermediate-size moa, buried in occupation deposit and immediately below human skeletons buried in top layer of clean sand. Tautuku, Otago ( $46^{\circ} 36^{\prime} \mathrm{S}$ Lat, $169^{\circ} 24^{\prime} \mathrm{E}$ Long). Coll. by L. Lockerbie. 
Mytilus shell, from deposit on sandhill slope, well above sealevel, covered by clean sand at Cannibal Bay, Otago (46 $29^{\prime} \mathrm{S}$ Lat, $169^{\circ} 45^{\prime} \mathrm{E}$ Long). Coll. by L. Lockerbie.

\section{Warehou Bay, Makara series}

Shells and wood from butt of palisade post from abandoned Maori occupation site at W headland of Warehou Bay, Makara, Wellington Coast $\left(41^{\circ}\right.$ 13’ S Lat, 174' 42' E Long), N160/269305. Coll. by J. W. Brodie. Comment: site abandoned before European arrival in district, and no account preserved in Maori tradition.

NZ-148. Shells

NZ-149. Wood

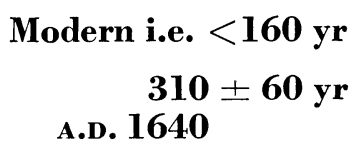

\section{Cape Campbell Concretion series}

Shell and matrix of a fossiliferous calcareous concretion dredged from $-420 \mathrm{ft}$ off Cape Campbell, Cook Strait $\left(41^{\circ} 21^{\prime} \mathrm{S}\right.$ Lat, $174^{\circ} 17^{\prime} \mathrm{E}$ Long). Coll. by J. W. Brodie. Comment: shells include abundant Chlamys delicatula, now very rare and small in Cook Strait but common in colder water to the $S$; they lived in a cold climate (Pantin, 1957).

\section{NZ-150. Shells of Chlamys delicatula \\ $19,500 \pm 1000$ 17,550 в.с. \\ NZ-151. Matrix of concretion

$$
\mathbf{2 7 , 5 0 0} \pm \mathbf{3 0 0 0}
$$$$
\text { 25,550 в.c. }
$$

\section{Sea Bed, Cook Strait series}

Shells from Cook Strait Narrows $\left(41^{\circ} 21^{\prime}\right.$ S Lat, $174^{\circ} 17^{\prime}$ E Long). Coll. by J. W. Brodie. Comment: shells were worn, bored, and stained, therefore processes giving appearance of age are rapid in Cook Strait.

\section{NZ-152. Ostrea sinuata shells \\ NZ-153. Venericardia shells \\ NZ-154. Chlamys shells
NZ-155. Various lamellibranchs outside Cloudy Bay

\section{NZ-156. Putaruru}

Carbon flecks from soil underlying the Tirau ash Putaruru-Rotorua Rd. 3.5 mi NE Putaruru ( $38^{\circ} 00^{\prime}$ S Lat, $175^{\circ} 49^{\prime}$ E Long), N66/308204. Coll. by C. A. Vucetich and D. Cross, N. Z. Soil Bur., DSIR. Comment: gives an older limit for the Tirau ash. 


\section{NZ-157. Taupo}

Charred wood 10 to 12 in. above Waimihia lapilli member and 8 to 10 in. below base of Taupo lapilli member, in the fossil soil next below that member. Taupo-Rotorua Rd. 0.25 mi NE of Kaimanawa Rd. ( $38^{\circ} 34^{\prime} \mathrm{S}$ Lat, $176^{\circ}$ 14' E Long), N94/695523. Coll. by J. Healy.

\section{NZ-158. Hastings}

$$
1760 \pm 80
$$

A.D. 190

Charcoal from a 10-ft bed in pumice pit on Pakowai Rd. 0.5 mi SSW of Longlands Rd., Hastings (39 $40^{\prime} \mathrm{S}$ Lat, $176^{\circ} 47^{\prime} \mathrm{E}$ Long), N134/212181. Coll. by T. L. Grant-Taylor. Comment: dates the Taupo Lapilli member (see NZ-1, 3, 4, etc.) center of origin $90 \mathrm{mi}$ W.

\section{NZ-159. Hastings}

$1750 \pm 80$

Wood from shrubs in growth position killed by partial burial in the pumice flood debris and consequent rise of watertable. Contained charcoal dated $1760 \pm 80 \mathrm{yr}$, NZ-158. From drain 1 chain $\mathrm{E}$ of unformed road between Irongate and Maraekakaho Rds., Hastings ( $39^{\circ} 39^{\prime} \mathrm{S}$ Lat, $176^{\circ} 47^{\prime} \mathrm{E}$ Long), N134/206194. Coll. by T. L. Grant-Taylor. Comment: date corresponds so closely with that of NZ-158 that the eruption, charring of trees and formation of a pumice flood must have been nearly contemporaneous.

\section{NZ-160. Fort Galatea}

$1300 \pm 80$

Wood from log of Podocarpus totara between Taupo pumice ash and Kaharoa ash from S side Rawhiti Rd. 6 mi NNW of Fort Galatea $\left(38^{\circ} 19^{\prime} \mathrm{S}\right.$ Lat, $176^{\circ} 40^{\prime}$ E Long), N86/121816. Coll. by R. J. Cameron, N. Z. Forest Service. Comment: fixes on maximum age of Kaharoa ash.

\section{NZ-161. Kinleith}

$$
1780 \pm 80
$$

A.D. 170

Wood from topmost member of Taupo pumice in L bank of sludge channel Kinleith Timber Mill $0.25 \mathrm{mi}$ from Mill ( $38^{\circ} 27^{\prime} \mathrm{S}$ Lat, $175^{\circ} 53^{\prime} \mathrm{E}$ Long), N84/371871. Coll. by J. Healy.

\section{NZ-162. Kinleith}

$$
\begin{array}{r}
1830 \\
\text { A.D. } 120
\end{array}
$$

Charcoal (small twigs and branches) from Taupo pumice, believed to be mudflow from sludge channel, Kinleith Timber Mill, R bank, $0.25 \mathrm{mi}$ from Mill ( $38^{\circ} 17^{\prime}$ S Lat, $173^{\circ} 53^{\prime}$ E Long), N84/371871.

\section{NZ-163. Atiamuri Road}

$$
\begin{array}{r}
1840 \\
\text { A.D. } 110
\end{array}
$$

Charcoal and twigs from dark gray pumice sand and rhyolite tuff and lapilli above Taupo pumice from pumice pit $\mathrm{W}$ side Taupo-Atiamuri Rd. (38 $28^{\prime} \mathrm{S}$ Lat, $176^{\circ} 04^{\prime} \mathrm{E}$ Long), N85/533645. Coll. by J. Healy.

\section{NZ-164. Atiamuri}

$$
\begin{array}{r}
1890 \\
\text { A.D. } 60
\end{array}
$$

Twigs from uppermost bed in Taupo pumice, in pumice pit $\mathrm{W}$ side of 
Taupo-Atiamuri Rd., $1 \mathrm{mi} \mathrm{N}$ of Maroa Corner $\left(38^{\circ} 28^{\prime} \mathrm{S}\right.$ Lat, $176^{\circ} 03^{\prime} \mathrm{E}$ Long). Coll. by J. Healy.

\section{NZ-165. Arapuni}

$1900 \pm 70$

A.D. 50

Small branches, roots, twigs and leaves overlying old soil at site of forest buried below pumice flood at spillway below Arapuni Dam $\left(38^{\circ} 04^{\prime} \mathrm{S}\right.$ Lat, $175^{\circ} 38^{\prime}$ E Long), N75/134136.

\section{NZ-166. Lake Okaro}

\section{A.D. 1110}

$\mathbf{8 4 0} \pm \mathbf{5 0}$

Wood of Leptospermum scoparium $70 \mathrm{yr}$ old in volcanic breccia at depth $20 \mathrm{ft}$ on $\mathrm{S}$ bank, Lake Okaro (38 $18^{\circ} \mathrm{S}$ Lat, $176^{\circ} 23^{\prime} \mathrm{E}$ Long), N85/853841. Coll. by D. Cross.

\section{NZ-167. Lake Tutaeinanga}

$3110 \pm 70$

Wood of partially decomposed log Dacrydium cupressimum $4 \mathrm{ft}$ below surface and 9 in. above Taupo ash bed, in altered andesitic ash erupted from site of Lake Ngapouri. Sample from pit $2 \mathrm{mi} \mathrm{W}$ of Waiotapu Hotel and near Lake Tutaeinanga $\left(38^{\circ} 20^{\prime} \mathrm{S}\right.$ Lat, $176^{\circ} 19^{\prime} \mathrm{E}$ Long), N85/783802.

\section{NZ-168. Pueto River}

$$
1900 \pm 60
$$

A.D. 50

Wood in dark brown peaty bed immediately below Hatepe Lapilli bed 36 $\mathrm{ft}$ below surface. In road cut on left bank of Pueto River at bridge on Broadlands-Taupo Rd. ( $38^{\circ} 37^{\prime} \mathrm{S}$ Lat, $176^{\circ} 16^{\prime}$ E Long), N94/723463. Coll. by C. G. Vucetich.

\section{NZ-169. Rainbow Mountain, Waiotapu}

A.D. 1050

$900 \pm 40$

Wood from outer few rings of trees lying directly on top of soil formed from Taupo pumice showers ca. 1800 yr ago. NZ-1, 3, 4, 158, 159, 161, etc. and covered by $20 \mathrm{ft}$ of hydrothermally altered andesite, $\mathrm{W}$ side of eroded gulch entering Lake Nahewa Crater, Rainbow Mt., Waiotapu $\left(38^{\circ} 19^{\prime} \mathrm{S}\right.$ Lat, $176^{\circ}$ 22' E Long), N85/828834. Coll. by E. F. Lloyd, N. Z. Geol. Surv. Comment: fixes maximum date of andesite.

\section{NZ-170. Tongariro National Park}

$$
\begin{array}{r}
1800 \\
\text { A.D. } 150
\end{array}
$$

Charred wood from 4 in. above base of rhyolitic pumiceous ash which is overlain by $10 \mathrm{in}$. of Ngaurohoe ash and underlain by $50 \mathrm{in}$. of Tongariro ash and lapilli. Tongariro Natl. Park-Wanganui Highway, $1 \mathrm{mi} \mathrm{S}$ of junction of Natl. Park-Taupo Rd. (39 $19^{\prime}$ S Lat, $175^{\circ} 24^{\prime}$ E Long), N11l/905769. Coll. by D. R. Gregg, N. Z. Geol. Sur. Comment: date is that of Taupo lapilli member.

\section{NZ-171. Whakamaru-Tihoi Road}

$2650 \pm 150$

700 в.с.

Branches, roots, and twigs, collected from near top of weathered ash shower. From cut on Whakamaru-Tihoi Rd. (38 $25^{\prime} \mathrm{S}$ Lat, $175^{\circ} 48^{\prime} \mathrm{E}$ Long), 
N84/283702. Coll. by D. Cross. Comment: sample appears to date host bed rather than overlying ash.

\section{NZ-172. Lake Rerewhakaitu}

$1800 \pm 100$

Carbonized root from Taupo ash Bretts Rd., near N shore Lake Rerewhakaitu (38 $17^{\prime} \mathrm{S}$ Lat, $176^{\circ} 30^{\prime}$ E Long), N84/958868. Coll. by C. G. Vucetich and D. Cross.

\section{NZ-173. Atiamuri}

$1750 \pm 50$

A.D. 200

Branches and twigs from basal $6 \mathrm{ft}$ of the coarse pumice member in a sequence of pumice-flood deposits carried down the Waikato River Road cut near Atiamuri State Hydro-electric Sta. ( $38^{\circ} 24^{\prime} \mathrm{S}$ Lat, $176^{\circ} 01^{\prime} \mathrm{E}$ Long), N85/496736. Coll. by J. Healy and B. N. Thompson.

\section{NZ-174. Atiamuri \\ $1800 \pm 100$}

Charcoal from base of pumice member, $6 \mathrm{ft}$ thick, underlying coarse pumice member (NZ-173). Road cut near Atiamuri State Hydro-electric Sta. ( $38^{\circ} 24^{\prime}$ S Lat, $176^{\circ} 01^{\prime}$ E Long), N85/496736. Coll. by J. Healy and B. N. Thompson.

\section{NZ-175. Wairakei}

$$
\begin{array}{r}
1850 \\
\text { A.D. } 100
\end{array}
$$

Small charred branches and twigs from rhyolite block member. Compares age of rhyolite block member with that of lapilli member. NZ-1, 3, 4, etc. Rotorua-Taupo Rd., 4 mi NE of Wairakei ( $38^{\circ} 36^{\prime} \mathrm{S} \mathrm{Lat}, 176^{\circ} 09^{\prime} \mathrm{E}$ Long), N94/618489. Coll. by J. Healy.

\section{NZ-176. Terraces Quarry, Taupo}

$$
1900 \pm 70
$$

Charcoal dust and fragments of organic material up to 0.25 in. in size, from top inch of Ash Member 9 (Hatepe lapilli) Rotorua Rd., 4 mi NE Wairakei ( $38^{\circ} 43^{\prime} \mathrm{S}$ Lat, $176^{\circ} 07^{\prime}$ E Long), N94/589347. Coll. by J. Healy.

NZ-177.

$$
2500 \pm 200
$$

550 в.C.

Charcoal fragments up to $0.5 \mathrm{in}$. in size, from top inch of Ash Member 11. From quarry on Napier Highway, $1.25 \mathrm{mi}$ SE of Terraces Hotel, Taupo $\left(38^{\circ}\right.$ $43^{\prime}$ S Lat, $176^{\circ} 07^{\prime}$ E Long), N94/589347. Coll. by J. Healy.

\section{NZ-178. Wairakei}

$2100 \pm 100$

150 в.с.

Partially carbonized dust and bits of organic material up to 0.5 in. diam coated with white sediment, from topmost 0.5 in. of Ash Member 9. (Hatepe lapilli). For locality see below, NZ-179.

NZ-179.

$$
\begin{aligned}
& 3420 \pm 70 \\
& 1470 \text { в.с. }
\end{aligned}
$$

Charred twigs and branches 0.25 to 1 in. from top of Ash Member 19 (Waimahia lapilli member). From top of first rise on access road to Bore 203 Wairakei (38 $38^{\prime} \mathrm{S}$ Lat, $176^{\circ} 05^{\prime}$ E Long), N94/560439. Coll. by J. Healy. 


\section{NZ-180. Hinemaiai River}

$3150 \pm 90$

1200 в.C.

Carbonized wood from charred $\log 2 \mathrm{ft}$ above base of a pumice breccia bed $60 \mathrm{ft}$ thick. R bank Hinemaiai River upstream from Pahikokura Creek, Taupo ( $38^{\circ} 53^{\prime}$ S Lat, $176^{\circ} 04^{\prime}$ E Long), N103/531143. Coll. by J. Healy.

\section{NZ-181. Okahukura Bush}

Modern, i.e. $<\mathbf{2 0 0}$

Wood from ash layer beneath flow from Te Mari volcanic vent. 4 chains from face of older Te Mari flow. Mangatetipua Stream in Okahukura Bush (39 08' S Lat, $175^{\circ} 40^{\prime}$ E Long), N112/150900. Coll. D. R. Gregg and E. F. Lloyd.

\section{NZ-182. Kaimanawa Road}

$2800 \pm 100$ 850 в.c.

Charcoal up to $0.25 \mathrm{in}$. from near center of layer of brown rhyolitic ash 3 to 5 in. thick 2 in. above Waimihia lapilli member. (NZ-179) from Rotorua Taupo Rd. $0.25 \mathrm{mi}$ NE of Kaimanawa Rd. corner $\left(38^{\circ} 34^{\prime} \mathrm{S}\right.$ Lat, $176^{\circ} 14^{\prime} \mathrm{E}$ Long), N94/695523. Coll. J. Healy.

\section{Pohokura Road series}

NZ-183. Charcoal 27 to 29 in. below surface

$$
\begin{aligned}
1800 & \pm 70 \\
\text { A.D. } 150 & \\
\mathbf{2 4 0 0} & \pm \mathbf{8 0} \\
\mathbf{4 5 0} \text { в.C. } &
\end{aligned}
$$

NZ-184. Charcoal 36 to 39 in. below surface

Pohokura Rd. 9 mi. NW of Tutira, Hawkes Bay $\left(39^{\circ} 07^{\prime}\right.$ S Lat, $176^{\circ} 49^{\prime}$ E Long), N114/247843. Coll. by H. S. Gibbs. Comment: samples in distinct beds of separate pumice sands, the lower immediately above the Waimihia lapilli member.

\section{NZ-185. Terraces Quarry Taupo}

$8850 \pm 1000$

Carbon flecks and soil from bed 5 in. thick immediately below the Waimihia lapilli member. Terraces Quarry $1.5 \mathrm{mi} \mathrm{SE}$ of Terraces Hotel, Taupo $\left(38^{\circ}\right.$ 43' S Lat, $176^{\circ} 49^{\prime}$ E Long), N94/589347. Coll. by C. A. Vucetich.

\section{NZ-186. Mangatawai Stream}

Nothofagus sp. leaves from lower 3 in. of unweathered andesitic ash 20 in. thick. The ash which may have come from Mt. Ngauruhoe, is separated from the overlying Taupo pumice by $13 \mathrm{in}$. of andesictic ash without leaves. The leaf bearing ash is a marker bed throughout $150 \mathrm{sq} \mathrm{mi}$. Cut on WaiouruTurangi Highway at bridge over Mangatawai Stream, 1 chain S of bridge on $\mathrm{S}$ side of road ( $39^{\circ} 09^{\prime} \mathrm{S}$ Lat, $175^{\circ} 46^{\prime}$ E Long). Coll. by D. R. Gregg.

\section{NZ-190. Blanche Bay, New Guinea}

$$
1190 \pm 60
$$

\section{A.D. 760}

Charcoal from the lower part of pyroclastic deposits which form the wall of Blanche Bay caldera. Tunnel Hill Rd., Blanche Bay, New Guinea $\left(04^{\circ} 11^{\prime}\right.$ S Lat, $152^{\circ} 10^{\prime} \mathrm{E}$ Long). Coll. by M. A. Reynolds, Vulcanological Observatory, 
Rabaul, New Guinea. Comment: sample dates latest catastrophic phase of activity.

NZ-191. Double Island Point Australia

Wood Podocarpus sp. in lignite (containing mangrove) at high-water mark. Beach front $4 \mathrm{mi} \mathrm{S}$ of Double Island Point, Australia $\left(26^{\circ} 07^{\prime} \mathrm{S}\right.$ Lat, $153^{\circ} 07^{\prime}$ E Long). Coll. J. E. Coaldrake, CSIRO, Brisbane.

NZ-192. Double Island Point, Australia

Wood Myrtaceous not Eucalyptus, probably Metrosideros, from bed of compact carbonaceous sand at high-water mark. Association suggests higher sealevel and warmer climate. Beach front $8 \mathrm{mi} \mathrm{S}$ of Double Island Point, Australia $\left(25^{\circ} 55^{\prime} \mathrm{S} \mathrm{Lat}, 153^{\circ} 07^{\prime} \mathrm{E}\right.$ Long). Coll. by J. E. Coaldrake.

NZ-193.

$39,000 \pm 3000$

Charcoal in sandy matrix $250 \mathrm{ft}$ above sealevel. Gives minimum age for Teewah Sands. For locality see NZ-194.

NZ-194.

37,050 в.c.

28,350 в.c.

Organic matter of cemented sand $10 \mathrm{ft}$ below hardpan of a former ground water podzol. Cliffs fronting beach $8 \mathrm{mi} \mathrm{S}$ of Double Island Point, Australia ( $26^{\circ} 06^{\prime}$ S Lat, $153^{\circ} 07^{\prime}$ E Long). Coll. by J. E. Coaldrake.

\section{NZ-195. Eucania, Australia}

$6270 \pm 120$

4320 в.c.

Mangrove peat from flat a few feet above high-water mark. Eucania near Babinda, Australia ( $17^{\circ} 16^{\prime} \mathrm{S}$ Lat, $145^{\circ} 56^{\prime}$ E Long). Coll. by R. M. Moore, CSIRO, Canberra.

NZ-196. Mildura, Australia

$5400 \pm 80$ 3450 в.с.

Wood from depth $35 \mathrm{ft}$ in a bore $83 \mathrm{ft}$ above sealevel. Physche Bend, Mildura, Australia (34, 15’ S Lat, $142^{\circ} 14^{\prime}$ E Long). Coll. by F. Penman, Irrigation Res. Sta., Merbein, Victoria.

NZ-197. Tomago, Australia

Shell (Anadara sp.) from bore 29A depth $60 \mathrm{ft}$ in Tomago sandbeds, Tomago, Australia $\left(32^{\circ} 50^{\prime} \mathrm{S}\right.$ Lat, $151^{\circ} 45^{\prime} \mathrm{E}$ Long). Coll. by E. E. K. Duncan, Hunter Dist. Water Board, Newcastle, Australia. Comment: places a younger limit on the marine transgression which laid down the Tomago sandbeds.

\section{Soils from Southeastern Australia}

Charcoal and wood fragments deposited with the soils of four different cycles. $K_{3}$ and $K_{0}$ from $34^{\circ} 51^{\prime} S$ Lat, $150^{\circ} 31^{\prime}$ E Long. $K_{2}$ and $K_{1}$ from $34^{\circ}$ $56^{\prime}$ S Lat, $150^{\circ} 35^{\prime}$ E Long. Coll. by C. B. F. Butler, CSIRO. Canberra.

NZ-198. $\quad \mathbf{K}_{3}$ cycle $29,000 \pm 800$ 27,050 в.с. 
NZ-199. $K_{2}$ cycle

NZ-200. $K_{1}$ cycle

A.D. 1560

$390 \pm 60$

modern $<120$

Modern $<\mathbf{2 0 0}$

\section{$\begin{array}{ll}\text { NZ-201. } & K_{0} \text { cycle } \\ \text { NZ-202. } & \text { West Pakistan }\end{array}$}

Charcoal associated with sherd in top $12 \mathrm{in}$., underlain by flint flakes and microliths of a mesolithic culture. Kapoto Rock Shelter Baluchistan $\left(28^{\circ} 47^{\prime}\right.$ N Lat, $66^{\circ} 35^{\prime}$ E Long). Coll. by H. Field, Peabody Mus., Cambridge, Mass. U.S.A. Comment: date places an upper limit on mesolithic occupation (Field, 1959).

\section{NZ-203. West Pakistan}

Modern $<\mathbf{2 0 0}$

Charcoal from occupation mound with plain sherds in upper meter which had been used as a Buddist Stupa $\left(26^{\circ} 44^{\prime} \mathrm{N}\right.$ Lat, $63^{\circ} 55^{\prime} \mathrm{E}$ Long). Coll. by H. Field. Comment: sample is from upper meter.

\section{NZ-204. New Guinea}

Modern $<100$

Carbonized wood from small tree trunk buried in a catastrophic eruption from Long Island Crater, New Guinea ( $5^{\circ} 20^{\prime} \mathrm{S}$ Lat, $147^{\circ} 13^{\prime} \mathrm{E}$ Long). Coll. by G. A. Taylor, Vulcanological Observatory, Rabaul, New Guinea.

\section{NZ-205. Lake Callabona, South Australia}

Presumed crop contents of Diprotodon, a giant herbivorous marsupial which became extinct in sub-recent time, presumably as the result of climate change. Orroroo, Lake Callabona, S Australia (29 $50^{\prime} \mathrm{S}$ Lat, $140^{\circ} 05^{\prime} \mathrm{E}$ Long). Coll. by D. Mawson, Univ. of Adelaide, S Australia.

NZ-206.

$$
6700 \pm 250
$$

\section{0 в.c.}

Dentine from lower-jaw teeth of Diprotodon. It had been believed that the "crop contents" NZ-205 were related to the animal whose teeth were dated NZ-206. Orroroo (29 $50^{\prime}$ S Lat, $140^{\circ} 05^{\prime}$ E Long). Coll. by D. Mawson.

\section{NZ-207. Keilor, Victoria}

$18,000 \pm 500$

Charcoal from aboriginal fireplace $5 \mathrm{ft} 9 \mathrm{in}$. below level of cranium from Keilor Cranium Quarry in Keilor Terrace where Dry Creek enters Maribyr. nong River, $1 \mathrm{mi} \mathrm{N}$ of Keilor, near Melbourne, Victoria (37 $52^{\prime} \mathrm{S}$ Lat, $144^{\circ}$ $50^{\prime}$ E Long). Coll. by E. D. Gill, Natl. Mus. of Victoria, Melbourne.

\section{NZ-215. Auckland}

$29,000 \pm 1500$

\section{7,050 в.с.}

Wood from outer portion of tree in growth position, in tuff covered by flows from Ihumatao volcanic center, $\mathrm{S}$ shore of Ihumatao, Mangere, Auckland ( $37^{\circ} 00^{\prime} \mathrm{S}$ Lat, $174^{\circ} 45^{\prime}$ E Long), N42/273427. Coll. by E. J. Searle, Auckland Univ. 


\section{NZ-216. Auckland}

$$
\begin{aligned}
& 28,000 \pm 1000 \\
& 26,050 \text { в.c. }
\end{aligned}
$$

Wood covered with ash probably originating from Three Kings volcanic center, and near margin of lavas from $\mathrm{Mt}$. Eden volcanic cone. In sewage tunnel, Woodside Rd., Mt. Eden, Auckland ( $36^{\circ} 53^{\prime}$ S Lat, $174^{\circ} 45^{\prime}$ E Long), N42/277504. Coll. by E. J. Searle. Comment: sample probably antedates Mt. Eden basalt.

\section{NZ-217. Auckland}

$28,000 \pm 1000$ 26,050 в.c.

Peat underlying Panmure basin tuff in terrace of Tamaki River, Auckland $\left(36^{\circ} 55^{\prime} \mathrm{S}\right.$ Lat, $174^{\circ} 52^{\prime}$ E Long), N42/382539. Coll. by E. J. Searle. Comment: result gives an older limit for volcanic activity at this center, and dates a constructional terrace.

\section{Takapuna series, Auckland}

$\begin{array}{llr}\text { NZ-218. Charred wood } & >42,000 \\ \text { NZ-219. Peat } & >40,000\end{array}$

Samples taken from peat bed underlying basalt and tuffs of Pupuke Volcano in Borough Council yard, corner Anzac St. and Tauhoroto Rd., Takapuna $\left(36^{\circ} 48^{\prime} \mathrm{S}\right.$ Lat, $174^{\circ} 46^{\prime} \mathrm{E}$ Long), N42/282679. Coll. by E. J. Searle. Comment: date places older limit on Pupuke volcanics and a younger limit on a low terrace on shore of Shoal Bay. (Searle, 1959b).

\section{Rangitoto series, Auckland}

Shells from ash-free sand (NZ-220) underlying Rangitoto ash. Charcoal from base of oven (NZ-221) in $8 \mathrm{ft}$ thick Rangitoto ash, W side Administration Bay, Motutapu Island, near Rangitoto Island ( $36^{\circ} 45^{\prime} \mathrm{S}$ Lat, $174^{\circ} 54^{\prime} \mathrm{E}$ Long), N38/421726. Coll. by R. N. Brothers, Auckland Univ. Comment: Rangitoto Island is a very young volcano whose activity is suggested by Maori tradition. Samples span period of activity. (Brothers and Golson, 1959).

\section{NZ-220. Motutapu Island, Auckland}

\section{NZ-221. Base of oven}

\section{NZ-222. Motutapu Island, Auckland}

$\mathbf{7 5 0} \pm \mathbf{5 0}$

A.D. 1200

A.D. 1670

$$
280 \pm 40
$$

$$
\mathbf{7 7 0} \pm \mathbf{5 0}
$$

Shells from ash free sand (NZ-220) underly Rangitoto ash charcoal from base of oven (NZ-221) in $8 \mathrm{ft}$ thick Rangitoto ash from W side Administration Bay, carbonized wood (NZ-222) underlying Rangitoto ash E side, Administration Bay, Motolapu Is. ( $36^{\circ} 45^{\prime} \mathrm{S}$ Lat, $174^{\circ} 54^{\prime} \mathrm{E}$ Long), N38/421726 and N38/423728. Coll. by R. N. Brothers, Auck. Univ. Comment: Rangitoto Is. is a very young volcano whose activity is suggested by Maori tradition samples span period of activity. 
NZ-223. Mt. Albert, Auckland

Charred branch beneath $15 \mathrm{ft}$ of sub-recent lava in Oakley Creek Quarry, Mt. Albert, Auckland ( $36^{\circ} 53^{\prime}$ S Lat, $174^{\circ} 42^{\prime}$ E Long), N42/225565. Coll. by W. E. Begbie; subm. by E. J. Searle.

NZ-224. Auckland

Carbonaceous soil below tuff, $55 \mathrm{ft}$ thick, from Onepoto tuff ring, in Bore

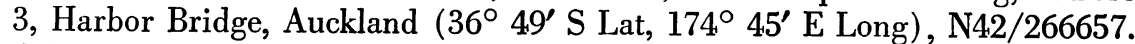
Coll. by E. J. Searle. Comment: date sets younger limit for volcanic activity at this center during a time of low sealevel.

\title{
NZ-225. Penrose, Auckland
}

$9000 \pm 160$ 7050 в.с.

Charcoal imbedded in basalt from Mt. Short volcano, Penrose, Auckland ( $36^{\circ} 55^{\prime} \mathrm{S}$ Lat, $174^{\circ}$ 49’ E Long), N42/335530. Coll. by J. A. Bartrum; subm. by E. J. Searle.

\section{NZ-226. Takapuna, Auckland}

Modern $<\mathbf{2 0 0}$

Wood from cavity in basalt on foreshore between Takapuna Beach and

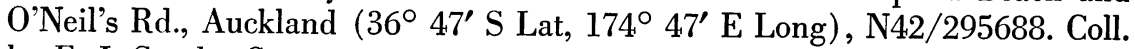
by E. J. Searle. Comment: apparently driftwood.

NZ-227. Takapuna, Auckland

Charcoal from cinders in $\mathrm{E}$ face of Smales Quarry, Takapuna, Auckland (36 $47^{\prime}$ S Lat, $174^{\circ} 45^{\prime}$ E Long), N42/277686. Coll. by E. J. Searle.

\section{Hauraki Gulf series}

\author{
HOLOCENE STUDIES
}

POSTGLACIAL SEALEVELS

\section{New Zealand}

Samples (NZ-265 to 273) collected to determine changes of younger postglacial sealevel. Samples NZ-269 and NZ-271 were collected $7 \mathrm{mi}$ N of the others and their projected position with respect to the bulk of the samples is given.

\section{NZ-265. Kaiaua and Miranda}

$$
\begin{array}{r}
980 \pm 60 \\
\text { A.D. } 970
\end{array}
$$

Shell $850 \mathrm{yd}$ from present ridge and $9 \mathrm{ft}$ above mean sealevel. Halfway between Kaiaua and Miranda (37 $09^{\prime} \mathrm{S}$ Lat, $175^{\circ} 18^{\prime} \mathrm{E}$ Long), N48/811245. Coll. by J. C. Schofield. Comment: dates sealevel of $0 \mathrm{ft}$.

\section{NZ-266. Miranda$$
1160 \pm 60
$$ \\ A.D. 790}

Shell $5 \mathrm{ft}$ above mean sealevel. Miranda Shell Bank $1 \mathrm{mi} \mathrm{N}$ of Miranda ( $37^{\circ} 10^{\prime} \mathrm{S}$ Lat, $175^{\circ} 19^{\prime} \mathrm{E}$ Long), N48/823220. Coll. by N. H. Taylor. Comment: position between NZ-265 and NZ-267 but exact height not established.

\section{NZ-267. Miranda}

$$
\begin{array}{r}
1540 \\
\text { A.D. } 410
\end{array}
$$

Shell 1270 yd from present ridge, $9 \mathrm{ft}$ above mean sealevel. Halfway be- 
tween Kaiaua and Miranda ( $37^{\circ} 09^{\prime}$ S Lat, $175^{\circ} 18^{\prime}$ E Long), N48/807244.

Coll. by J. C. Schofield. Comment: dates sealevel of $+1.5 \mathrm{ft}$.

NZ-268. Miranda

$1760 \pm 70$

Shell $1590 \mathrm{yd}$ from present ridge, $10.5 \mathrm{ft}$ above mean sealevel. Halfway between Kaiaua and Miranda ( $37^{\circ} 09^{\prime}$ S Lat, $175^{\circ} 18^{\prime}$ E Long), N48/804243. Comment: dates a sealevel of $+0.5 \mathrm{ft}$.

\section{NZ-269. Kaiaua}

$\mathbf{2 3 7 0} \pm \mathbf{7 0}$

Shell $113 \mathrm{yd}$ from present ridge, $2 \mathrm{ft}$ above mean sealevel. Equivalent position in main sequence $1840 \mathrm{yd}, 3.5 \mathrm{mi} \mathrm{N}$ of Kaiaua near old gravel pit ( $37^{\circ} 04^{\prime}$ S Lat, $175^{\circ} 18^{\prime}$ E Long), N48/806353. Coll. by J. C. Schofield. Comment: dates sealevel of $-1.0 \mathrm{ft}$.

\section{NZ-270. Miranda}

$2730 \pm 70$

Shell $90 \mathrm{yd}$ inland from NZ-269, $3 \mathrm{ft}$ above mean sealevel. Halfway between Kaiaua and Miranda (37 $09^{\prime}$ S Lat, $175^{\circ} 18^{\prime}$ E Long), N48/799242. Coll. by J. C. Schofield.

\section{NZ-271. Miranda}

$\mathbf{3 1 5 0} \pm \mathbf{8 0}$

Shell $2130 \mathrm{yd}$ from present ridge, $11 \mathrm{ft}$ above mean sealevel. Halfway between Kaiaua and Miranda ( $37^{\circ} 04^{\prime} \mathrm{S}$ Lat, $175^{\circ} 18^{\prime} \mathrm{E}$ Long), N48/806353. Coll. by J. C. Schofield. Comment: dates a sealevel of $+4 \mathrm{ft}$.

\section{NZ-272. Miranda}

$3900 \pm 90$ 1950 в.c.

Shell $2610 \mathrm{yd}$ from present ridge, $16 \mathrm{ft}$ above mean sealevel. Halfway between Kaiaua and Miranda (37 $09^{\prime}$ S Lat, $175^{\circ} 17^{\prime}$ E Long), N48/794240. Coll. by J. C. Schofield. Comment: dates a sealevel of $+7 \mathrm{ft}$.

NZ-273. Miranda

Peat, behind ridge of NZ-272, halfway between Kaiaua and Miranda $\left(37^{\circ}\right.$ 09’ S Lat, $175^{\circ} 17^{\prime}$ E Long), N48/794240. Coll. by J. C. Schofield.

\section{Christchurch Sealevel series}

The following shell samples (NZ-274 to 277) were collected from a well one mile inland from the coast. The faunas change from estuarine in NZ-276 to include open-water species in NZ-274. The faunas show sealevel rising faster than deposition, with the shoreline moving westward. Tectonic movement is not expected.

\section{NZ-274. Christchurch}

$\mathbf{5 5 2 0} \pm \mathbf{7 0}$ 3570 в.с.

Shell depth $51 \mathrm{ft}$ below sealevel $\left(43^{\circ} 30^{\prime} \mathrm{S}\right.$ Lat, $172^{\circ} 43^{\prime} \mathrm{E}$ Long), S84/ 072592. Coll. by R. P. Suggate.

\section{NZ-275. Christchurch}

$7780 \pm 80$ 5830 в.с.

Shell depth 74, ft below sealevel, Palmers Rd., Pumping Sta., Christchurch (43 $30^{\prime}$ S Lat, $172^{\circ} 43^{\prime}$ E Long), S84/072592. Coll. by R. P. Suggate. 


\section{NZ-276. Christchurch}

$8530 \pm 110$

6580 в.с.

Shell depth $87 \mathrm{ft}$ below sealevel. Palmers Rd., Pumping Sta., Christchurch ( $43^{\circ} 30^{\prime}$ S Lat, $172^{\circ} 43^{\prime}$ E Long), S84/072595. Coll. by R. P. Suggate.

\section{NZ-277. Christchurch}

$5270 \pm 80$ 3220 в.c.

Peat from $2 \mathrm{ft}$ below present sealevel and probably overlain by estuarine silt. Railway excavation Christchurch $5 \mathrm{mi}$ W of coast $\left(43^{\circ} 33^{\prime} \mathrm{S}\right.$ Lat, $172^{\circ}$ 39' E Long), S84/010549. Coll. by R. P. Suggate.

\section{NZ-278. Upolu I, Western Samoa}

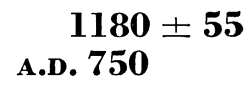

Coral sand at base of Tafagamanu Sand, $5 \mathrm{ft}$ above sealevel at Tafagamanu Village, Upolu I, Western Samoa ( $13^{\circ} 57^{\prime} \mathrm{S}$ Lat, $171^{\circ} 58^{\prime} \mathrm{W}$ Long). Coll. by B. L. Wood and D. Kear, N. Z. Geol. Surv. Comment: Tafagamanu Sand probably represents the accumulation of many hundreds of years of sealevels slightly higher than the present.

\section{NZ-279. Geelong Victoria}

Shells from emergent marine shell bed overlying bored freshwater Lara Limestone. From R bank Hovells Creek where crossed by Princes Highway near Geelong, Melbourne ( $38^{\circ} 03^{\prime} \mathrm{S}$ Lat, $144^{\circ} 25^{\prime} \mathrm{E}$ Long). Coll. by E. D. Gill.

\section{NZ-280. Byrones Creek, Queensland}

$3720 \pm 85$

1770 в.c.

Mangrove $\log$ in marine mud $9 \mathrm{ft}$ above present mangrove level. Dates a sealevel higher than the present. Bed of Byrnes Creek, near Babinda Queensland ( $17^{\circ} 15^{\prime} \mathrm{S}$ Lat, $145^{\circ} 55^{\prime} \mathrm{E}$ Long). Coll. by L. J. Webb, Botany Dept., Brisbane Univ., Queensland.

\section{NZ-281. Swan River Valley}

$9850 \pm 130$

\section{0 в.с.}

Freshwater peat from $68 \mathrm{ft}$ below sealevel in the valley of the Swan River, now submerged maximum of $140 \mathrm{ft}$. Narrows Bore, Swan River Valley, Western Australia ( $31^{\circ} 57^{\prime} \mathrm{S}$ Lat, $115^{\circ} 51^{\prime}$ E Long). Coll. by D. M. Churchill, Botany Depart., Univ. of Western Australia.

\section{NZ-282. Swan River Valley}

$7470 \pm 120$

5520 в.c.

Fossil marri (Eucalyptus calophylla) fruits, bark, wood and twigs, apparently in situ, from depth of 48 to $50 \mathrm{ft}$ and overlain by black shelly estuarine sands. Narrows Bore, Swan River Valley, Western Australia $\left(31^{\circ} 57^{\prime} \mathrm{S}\right.$ Lat, $115^{\circ} 51^{\prime}$ E Long). Coll. by D. M. Churchill. Comment: marri probably killed by rising sea so that sample dates a low sealevel.

\section{Coral series, West Africa}

Ahermatypic corals Dendrophyllia ramea and Madracis asperula from coral banks on the surface of trangressive Holocene sands. Banks are no longer 
living and their depth when alive cannot be deduced with certainty. Niger delta $\left(03^{\circ} 57^{\prime} \mathrm{N}\right.$ Lat, $06^{\circ} 58^{\prime}$ E Long). Coll. by J. R. L. Allen, Univ. Reading, England.

NZ-283. depth of $270 \mathrm{ft}$

NZ-284. depth of $312 \mathrm{ft}$

$$
2920 \pm 100
$$$$
970 \text { в.c. }
$$

$3885 \pm 125$ 2075 в.c.

Comment: if the banks formed at a level other than the present one compaction or isostatic or tectonic sinking must have occurred.

\section{HOLOCENE GENERAL}

\section{New Zealand}

\section{Ruahine Ranges series, North Island}

Samples collected for pollen analysis and dating to determine variations in recent climate and ages of two prominent pumice horizons. The pollen profiles are not yet completed.

\section{NZ-285.}

Wood (Dacrydium biforme) in fine uniform peat. Wood was apparently buried by creep of peat on a $10^{\circ}$ slope.

NZ-286.

$$
1040 \pm 50
$$

\section{A.D. 910}

Wood (Dacrydium biforme) from peat now dominated by red tussock. Depth 12 in., 3 in. above Taupo pumice horizon.

NZ-287.

$$
2800 \pm 80
$$

Wood (Dacrydium biforme) 7 in. below NZ-286 and 3 to 4 in. below Taupo pumice horizon. Depth 19 in.

\section{NZ-288.}

$$
\mathbf{2 8 0 0} \pm \mathbf{2 5 0}
$$

950 в.c.

Peat from pumice lapilli slightly below NZ-289. Depth 24 to $27 \mathrm{in}$. Sample should have been the same age as NZ-289. Roots from above appear to have affected the date which is therefore only minimum for this ash shower.

\section{NZ-289.}

$$
\begin{aligned}
& 3400 \pm 100 \\
& 1550 \text { B.C. }
\end{aligned}
$$

Large log of Dacrydium biforme 5 in. below NZ-287 with layer of grass overlying bank and in contact with layer of Waimihia lapilli, 2.5 to $3 \mathrm{in.}$ thick, which rises over it without change in thickness. Depth 20 to $24 \mathrm{in}$. Places an older limit on the Waimihia lapilli.

\section{NZ-290.}

Log (Dacrydium biforme) 5 in. below NZ-289. Probing of bog indicated greatest concentration of wood at this level, although wood abundant both 
above and below. Ratios of ages and depths show increased rate of deposition towards this level. Above sequence NZ-285 through NZ-290 $0.25 \mathrm{mi}$ due E of Ikawatea (Trig. E $4596 \mathrm{ft})$ from strip of bog at $4550 \mathrm{ft}\left(39^{\circ} 36^{\prime} \mathrm{S}\right.$ Lat, $176^{\circ}$ 16' E Long). Coll. by N. L. Elder, N. Z. Forest Service, Napier.

NZ-291.

$2100 \pm 60$

150 в.C.

Wood, probably root, below Taupo ash which rises over it. Group of tarns at $4580 \mathrm{ft} 10 \mathrm{yd} \mathrm{NW}$ of site $\left(39^{\circ} 36^{\prime} \mathrm{S}\right.$ Lat, $\left.176^{\circ} 16^{\prime} \mathrm{E} \mathrm{Long}\right)$. Coll. by N. L. Elder.

NZ-292.

$3400 \pm 90$

1550 в.C.

Wood (Dacrydium biforme) on margin of wind-blow in sandy peat. In wind-eroded hollow at depth of $10.5 \mathrm{ft} 0.25 \mathrm{mi} \mathrm{N}$ of Ohawai (Trig. 81, 4,485 ft) $\left(39^{\circ} 36^{\prime} \mathrm{S}\right.$ Lat, $176^{\circ} 16^{\prime} \mathrm{E}$ Long $)$. Coll. by N. L. Elder.

\section{NZ-293. Manawatu Wellington}

$\mathbf{8 5 5} \pm \mathbf{5 0}$

Tree trunk, rooted in place beneath $9 \mathrm{ft}$ of wind blown Himatangi sand. Kernshaw's Farm, Taikora, Manawatu $\left(40^{\circ} 22^{\prime} \mathrm{S}\right.$ Lat, $174^{\circ} 58^{\prime} \mathrm{E}$ Long $)$, N148/883334. Coll. by J. D. Cowie, N. Z. Soil Bur., Palmerston North. Comment: gives rate of accumulation of the sand, $8 \mathrm{mi}$ inland from coast.

NZ-294. Naike

$980 \pm 25$

A.D. 970

Kauri (Agathis australis) wood from stump that has altered Hamilton Ash, but not Mairoa Ash. Gives rates of changes in andesitic ash induced by Kauri ( $37^{\circ} 32^{\prime}$ S Lat, $174^{\circ} 58^{\prime}$ E Long), N55/475774. Coll. by N. H. Taylor.

\section{NZ-295. Cook Strait}

$615 \pm 75$

A.D. 235

Solitary corals (Flabellum), collected to determine whether specimens represent living forms or are excavated fossils. From Dominion Museum collection No. 180 from Cook Strait Canyon S of Palliser Bay at $900 \mathrm{ft}\left(41^{\circ} 28^{\prime}\right.$ S Lat, $176^{\circ} 50^{\prime}$ E Long). Subm. by D. F. Squires, Amer. Mus. Nat. Hist., New York. Comment: specimens could have died shortly before collection.

\section{NZ-296. Waiho River, Westland}

$$
1690 \pm 60
$$

$$
\text { A.D. } 260
$$

Wood, underlain by $6 \mathrm{ft}$ and overlain by $20 \mathrm{ft}$ of outwash gravel of a young glacial advance. Westland, E bank Waiho River 3 chain $\mathrm{N}$ of swing bridge on track from Peters Pool to Callery Gorge $\left(42^{\circ} 25^{\prime} \mathrm{S}\right.$ Lat, $170^{\circ} 11^{\prime} \mathrm{E}$ Long). Coll. by R. P. Goldthwait, Dept. of Geol., Ohio State Univ.

\section{NZ-297. Granite Creek}

$8350 \pm 135$

6400 в.c.

Wood from sand and silt $32 \mathrm{ft}$ thick and $20 \mathrm{ft}$ above creek level. Pollen analysis (W. F. Harris) shows a moist climate possibly warmer than present. Deposit formed during retreat of ice from maximum of Kumara III glacial 
substage Granite Ck. 6 chain upstream from road bridge $\left(42^{\circ} 27^{\prime} \mathrm{S}\right.$ Lat, $171^{\circ}$ $47^{\prime}$ E Long), S45/250882. Coll. by F. E. Bowen, N. Z. Geol. Sur.

\section{Palmer Road series, Westland}

Peat samples from bog behind a late last glacial moraine. SE margin of the bog is a postglacial fault scarp. Peat is light brown and fibrous at the top and dark brown, humified at the base, with a break marked by a mat of Nothofagus cliffortioides leaves and wood at $100 \mathrm{~cm}$. Rate of accumulation has been uniform. Pollen analysis by N. T. Moar shows shrub and small tree vegetation at the base replaced by beech forest. Palmer Rd., Westland $0.25 \mathrm{mi} \mathrm{S}$ of crooked Mary Creek about halfway along ditch dug to drain bog $\left(42^{\circ} 24^{\prime}\right.$ S Lat, $172^{\circ} 08^{\prime}$ E Long), S46/568944. Coll. by R. P. Suggate.

NZ-298. $38-42 \mathrm{~cm}$

NZ-299. $68-72 \mathrm{~cm}$

NZ-300. $\quad 93-97 \mathrm{~cm}$

\section{NZ-301. 118-122 cm}

\section{NZ-302. 138-142 cm}

\section{NZ-303. Shearers Swamp, S Westland}

Peat from basal layer of swamp formed behind a beach ridge. Dates commencement of swamp formation. Shearers Swamp, S Westland, 4 chain from $\mathrm{E}$ side in new road cut $\left(42^{\circ} 55^{\prime} \mathrm{S}\right.$ Lat, $170^{\circ} 46^{\prime} \mathrm{E}$ Long). Coll. by N. T. Moar.

\section{NZ-304. Porters Pass, Canterbury}

Charcoal from trees at depth of 0 to $8 \mathrm{in}$. below A horizon dates former widespread forest in area now vegetated by Danthonia tussock and Dracophyllum scrub gives older limit for destroying fire. Porters Pass Canterbury SE side of main ridge at $3500 \mathrm{ft}\left(43^{\circ} 18^{\prime} \mathrm{S}\right.$ Lat, $\left.171^{\circ} 45^{\prime} \mathrm{E} \mathrm{Long}\right), \mathrm{S} 74 / 218857$. Coll. by B. P. J. Molloy, Dept. of Agriculture, Christchurch.

\section{Christchurch Formation series}

Samples from the Christchurch formation with a pollen flora (N. T. Moar) suggesting an environment moister than the present, perhaps because of higher ground-water table.

\section{NZ-305. Hills Road}

$2040 \pm 60$

90 в.c.

Peat 12 in. thick at depth $4 \mathrm{ft}$. Pollen indicates Podocarp forest with $P$. spicatus and $P$. dacrydiodes common flora similar to younger NZ-86 (940 \pm 
70 yr) corner Hills Rd. and Edgeware Rd., Christchurch $\left(43^{\circ} 31^{\prime} \mathrm{S}\right.$ Lat, $172^{\circ}$ 39' E Long), S84/018584. Coll. by N. T. Moar.

\section{NZ-306. Gloucester Street \\ $3750 \pm 80$ \\ 1800 в.C.}

Peat from swamp forest with dominant Podocarp pollen, corner Gloucester St. and Cambridge Terrace, Christchurch $\left(43^{\circ} 32^{\prime} \mathrm{S}\right.$ Lat, $172^{\circ} 38^{\prime}$ E Long), S84/001564. Subm. by N. T. Moar.

\section{NZ-30\%. Conference Street}

$3160 \pm 808$

1210 в.c.

Peat with Podocarp flora corner of Conference and Durham Sts., Christchurch $\left(43^{\circ} 31^{\prime}\right.$ S Lat, $172^{\circ} 38^{\prime}$ E Long), S84/002572. Subm. by N. T. Moar.

$$
\text { AGGRADATION OF CHRISTCHURCH FORMATION }
$$

\section{Christehurch series}

The following give rates of Postglacial aggradation of alluvium in the Christchurch area.

\section{NZ-308. Ilam Road, NW Christchurch}

$$
\begin{aligned}
& 6980 \pm 100 \\
& 5030 \text { в.C. }
\end{aligned}
$$

Peat from $2 \mathrm{ft}$ above sealevel, $40 \mathrm{ft}$ below surface, at base of Christchurch formation. Dates beginning of local aggradation by Waimakariri River, Canterbury Univ., Ilam Rd., Fendalton $\left(43^{\circ} 32^{\prime}\right.$ S Lat, $172^{\circ} 35^{\prime}$ E Long), S84/ 955572. Coll. by R. P. Suggate.

\section{NZ-309. Council Yard, NW Christchurch}

$$
4660 \pm 90
$$

2710 в.c.

Peat from Christchurch Formation, depth $18 \mathrm{ft}$, showing aggradation had reached $15 \mathrm{ft}$ above sealevel at this time. Waimairi County Council Yard, Jeffereys Rd., Fendalton (43 $31^{\prime}$ S Lat, $172^{\circ} 36^{\prime}$ E Long), S84/970585. Coll. by B. W. Collins, Dept. Sci. Ind. Res., Wellington.

\section{Southwest Christchurch}

\section{$\begin{array}{ll}\text { NZ-310. Council Well, SW Christchurch } & 2990 \pm 110 \\ & 1040 \text { B.c. }\end{array}$}

Wood from gravel, depth $35 \mathrm{ft}$. Aggradation had reached $10 \mathrm{ft}$ above sealevel at this time. Christchurch City Council Well, Spreydon School, Lincoln Rd. ( $43^{\circ} 33^{\prime}$ S Lat, $172^{\circ} 35^{\prime}$ E Long), S84/964535. Coll. by L. E. Oborn, N. Z. Geol. Surv.

\section{NZ-311. Sparks Road, SW Christchurch}

$2460 \pm 70$

Wood from gravel, depth $5 \mathrm{ft}$. Aggradation had reached $30 \mathrm{ft}$ at this time. Sparks Rd. Bridge, Christlhurch $\left(43^{\circ} 35^{\prime} \mathrm{S}\right.$ Lat, $172^{\circ} 37^{\prime} \mathrm{E}$ Long), S84/982521. Coll. by B. W. Collins.

\section{NZ-312. Woolston Park, SW Christchurch}

$$
735 \pm 55
$$

\section{A.D. 1215}

Wood in estuarine silt, depth $9 \mathrm{ft} ; 2 \mathrm{ft}$ below sealevel, sewer excavation Woolston Park, Christchurch ( $43^{\circ} 33^{\prime}$ S Lat, $172^{\circ} 40^{\prime}$ E Long), S84/037543. Coll. by B. W. Collins. 


\section{Sub-Antarctic Islands}

\section{NZ-313. Auckland Island}

Peat $5 \mathrm{ft}$ above sealevel and $2 \mathrm{ft}$ below surface. Dates commencement of peat formation. Auckland Island, head of first bay to NNW of Ranui Cove, Port Ross $\left(50^{\circ} 32^{\prime}\right.$ S Lat, $166^{\circ} 13^{\prime}$ E Long). Coll. by M. Leamy, N. Z. Soil Bur.

Macquarie Island series

NZ-314.

$$
\begin{aligned}
& 7600 \pm 160 \\
& 5650 \text { в.c. }
\end{aligned}
$$

Basal peat, depth $6 \mathrm{ft}$ overlying bedrock and underlying gravel. Valley of Caroline Cove between Petrel Peak and Mt. Haswell alt ca. $7000 \mathrm{ft}\left(54^{\circ} 45^{\prime} \mathrm{S}\right.$ Lat, $158^{\circ} 49^{\prime}$ E Long). Comment: gives minimum estimate of time when S end of Macquarie Island was substantially ice free.

\section{NZ-315.}

$$
\text { A.D. } 440
$$$$
1510 \pm 55
$$

Basal peat depth $10 \mathrm{ft} 400 \mathrm{yd}$ up valley of Nuggets Creek near Nuggets Point, E coast Macquarie Island, alt $20 \mathrm{ft}$ ( $54^{\circ} 31^{\prime} \mathrm{S}$ Lat, $158^{\circ} 58^{\prime} \mathrm{E}$ Long).

\section{NZ-316.}

Peat depth $11 \mathrm{ft}$ back of raised beach terrace Eagle Point E Coast Macquarie Island (54. $30^{\prime} \mathrm{S}$ Lat, $158^{\circ} 54^{\prime} \mathrm{E}$ Long). Comment: appears to date only upper portion of covering sediments. Coll. by A. B. Costin, Div. of Plant Industries CSIRO Canberra.

\section{AUSTRALIA}

\section{Mt. Kosciusko series, Australia}

\section{NZ-317. Club Lake}

Peat from base of Carex swamp $12 \mathrm{ft}$ thick, overlying clay in cirque of Club Lake, Carruthers Peak alt $7000 \mathrm{ft}$, Mt. Kosciusko Plateau $\left(36^{\circ} 25^{\prime} \mathrm{S}\right.$ Lat, $148^{\circ} 18^{\prime}$ E Long). Comment: gives minimum age of current period of peat formation.

\section{NZ-318. Blue Lake}

Peat from top of Carex swamp, buried by mineral soils. Cirque above Blue Lake between Carruther's Peak and Mt. Twynam alt $7200 \mathrm{ft}\left(36^{\circ} 24^{\prime}\right.$ S Lat, $148^{\circ} 19^{\prime} \mathrm{E}$ Long). Coll. by A. B. Costin. Comment: date suggests burial probably caused by grazing activities.

\section{NZ-319. Angus River}

Charcoal, from alluvium of Angus Plains soil association, which is younger than the Bremner Plains association, (without E-W dunes) and the Milang combination (with E-W dunes) $\mathrm{N}$ bank. Angus Rd., $5 \mathrm{mi} \mathrm{N}$ of Milang, $4 \mathrm{mi}$ 
from Lake Alexandrina ( $35^{\circ} 20^{\prime} \mathrm{S}$ Lat, $\left.139^{\circ} 00^{\prime} \mathrm{E} \mathrm{Long}\right)$. Coll. by C. G. Stephens and C. Y. de Muoy, Div. of Soils, CSIRO, Adelaide. Comment: date adds to evidence that dune building ceased before the recent arid cycle.

\section{Boggy Lake series}

Samples from the humified peats of Boggy Lake, Western Australia that show climate changes in the upper postglacial $\left(35^{\circ} 00^{\prime} \mathrm{S}\right.$ Lat, $116^{\circ} 37^{\prime} \mathrm{E}$ Long). Coll. by D. M. Churchill.

\section{NZ-320. 2 m}

$\mathbf{2 4 5 0} \pm \mathbf{8 0}$

500 в.с.

Peat, highly humified; depth $2 \mathrm{~m}$ from $\mathrm{W}$ Australian Pollen Zone IV above Transition zone from Eucalyptus colophylla-Bankasia-Agonis Scrub of the subboreal to open heath of the Subatlantic.

\section{NZ-321. $3 \mathrm{~m}$}

$$
\begin{aligned}
& 3250 \pm 80 \\
& 1300 \text { в.c. }
\end{aligned}
$$

Sedge peat, highly humified, depth $3 \mathrm{~m}$. From W Australian Pollen Zone II/III, characterized by Eucalyptus colophylla maxima of the subboreal.

\section{NZ-322. $4 \mathrm{~m}$}

$$
4550 \pm 100
$$

2600 в.c.

Peat, humified, depth $4 \mathrm{~m}$, from base of $\mathrm{W}$ Australian Pollen Zone II, characterized by the commencement of the sharp increase in Eucalyptus colophylla during the early Subboreal.

NZ-323. Perth, Western Australia

Melaleuca stump, $2 \mathrm{ft} 6 \mathrm{in}$. diam, exposed by erosion of dunes. Present vegetation is coastal heath. Sample demonstrates striking floral change in modern times. $1 \mathrm{mi}$ S of City Beach, Perth $\left(31^{\circ} 55^{\prime} \mathrm{S}\right.$ Lat, $115^{\circ} 45^{\prime} \mathrm{E}$ Long $)$. Coll. by D. M. Churchill.

\section{NZ-324. Rottnest Island}

$$
7090 \pm 115
$$$$
6140 \text { в.C. }
$$

Blackboy stump, (Xanthorhoea sp.) (W Australian mus. No. G 9066) depth $19 \mathrm{ft}$. Rottnest Island off Fremantle Western Australia $\left(32^{\circ} 00^{\prime} \mathrm{S}\right.$ Lat, $115^{\circ} 30^{\prime} \mathrm{E}$ Long). Genus not recorded in early historical accounts of the flora; its local extinction is attributed to desiccation during the Hypsithermal maximum. Coll. by D. M. Churchill.

\section{Dead Seas series}

Two (NZ-325, NZ-326) samples of carbonaceous material, in silty and sandy clay, above and below a body of rock salt, buried in lacustrine sediments, at $\mathrm{S}$ end of S Basin, Dead Sea $\left(31^{\circ} 09^{\prime} \mathrm{N}\right.$ Lat, $35^{\circ} 27^{\prime} \mathrm{E}$ Long $)$. A third sample, NZ-327 ( $31^{\circ} 12^{\prime} \mathrm{N}$ Lat, $35^{\circ} 22^{\prime}$ E Long), from a submerged fossil forest exposed by $5 \mathrm{~m}$ fall of lake level in 1957-61. Coll. by D. Neev, Geol. Surv. of Israel.
NZ-325. 0 to $6.2 \mathrm{~m}$ sandy clay $(6.22$ to 8.05$) \mathrm{m}$ rock salt $4410 \pm 320$ 
NZ-326. 8.05 to $30.00 \mathrm{~m}$ silty clay

NZ-327. Wood from submerged forest
$9580 \pm 150$

7630 в.с.

modern, i.e. $<\mathbf{2 0 0}$

$930 \pm 165$

A.D. 1020

\section{NZ-328. Dead Sea}

Plant remains, from beneath $200 \mathrm{~m}$ of varved sediments and on top of thin salt layer. Water depth $29 \mathrm{~m}$, Dead Sea $\left(31^{\circ} 19^{\prime} \mathrm{N}\right.$ Lat, $35^{\circ} 25^{\prime} \mathrm{E}$ Long $)$. Coll. by D. Neev. Comment: in other nearby cores superincumbent sediments were thicker. Age is minimum for salt. Below depth of $40 \mathrm{~m}$ Dead Sea water is dense and does not mix with the shallower water. The salt layer could have been formed while lake level was falling or rising from the $40 \mathrm{~m}$ level. Present data support view that it was deposited during lake rise.

PLEISTOCENE STUDIES

\section{New Zealand}

\section{NZ-329. Ngaruawahia}

$$
16,300 \pm 270
$$

$$
\text { 14,350 в.с. }
$$

Fossilized herbs, interbedded with sediments of Hinuera Pumiceous Aggradation Terrace, $8 \mathrm{ft}$ below terrace surface. From cut on Ngaruawahia-Glen Massey Rd. $1.5 \mathrm{mi}$ from Ngaruawahia and $0.25 \mathrm{~m} \mathrm{~N}$ of Te Puroa Rd. $\left(37^{\circ}\right.$ 41' S Lat, $175^{\circ} 06^{\prime}$ E Long), N56/619603. Coll. by D. Kear and J. C. Schofield.

\section{NZ-330. Cambridge}

$20,000 \pm 500$

Peat from pumice beds of Hinuera Aggradation Terrace, $35 \mathrm{ft}$ below surface. Dates a break in building of terrace. Cambridge-Rotorua Highway; first cut $\mathrm{E}$ of Cambridge Golf Links ( $37^{\circ} 54^{\prime} \mathrm{S}$ Lat, $175^{\circ} 30^{\prime} \mathrm{E}$ Long), N66/ 003343. Coll. by H. W. Wellman and D. Kear.

\section{NZ-331. Opunake}

$34,400 \pm 1500$

32,450 в.c.

Stem of shrub from peat lens between 7th and 8th lahar deposits from cliff top, $50 \mathrm{ft}$ below Opunake surface. Opunake Beach, $200 \mathrm{yd}$ WNW from Hihiwera Stream $\left(39^{\circ} 28^{\prime}\right.$ S Lat, $173^{\circ} 51^{\prime}$ E Long), N118/445428. Coll. by T. L. Grant-Taylor. Comment: dates second major episode of ring-plain formation since last interglacial. Pollen analysis (W. F. Harris) determines climate at sealevel as similar to that now on Mt. Egmont at 4,000 ft.

NZ-332. Wellington

Peat alt 99 to $100 \mathrm{ft}$ test bore in reclaimed area, Evans Bay, Wellington City $\left(41^{\circ} 19^{\prime} \mathrm{S}\right.$ Lat, $174^{\circ} 48^{\prime} \mathrm{E}$ Long), N164/364183. Coll. by M. T. Te Punga.

\section{Hauraki Plains series}

NZ-333. Piako Swamp

$$
5370 \pm 90
$$

3420 в.c.

Peat immediately below a pumice horizon and with a pollen flora suggesting transition from period of greatest postglacial warmth seen in this pro- 
file. Dates floral transition at Piako Swamp-Hauraki Plains $\left(37^{\circ} 24^{\prime} \mathrm{S}\right.$ Lat, $175^{\circ} 34^{\prime}$ E Long), N53/070937. Coll. by W. F. Harris and J. C. Schofield.

\section{NZ-334. Piako Swamp}

$11,900 \pm 750$

9950 в.с.

Peat from depth $10.25 \mathrm{~m}$. Postdates a climatic amelioration representing the last large climatic fluctuation in this area $\left(37^{\circ} 25^{\prime} \mathrm{S}\right.$ Lat, $175^{\circ} 31^{\prime} \mathrm{E}$ Long), N53/103914. Coll. by W. F. Harris and J. C. Schofield.

\section{Wallaceville series}

NZ-335. Wallaceville Swamp

NZ-336.

$$
\begin{array}{rl}
1420 & \pm 60 \\
\text { A.d. } 530 & 1750 \\
1760 \\
\text { A.D. } 200
\end{array}
$$

Peat, from top and base of Gleichenia fern horizon dating a decrease in wetness of the swamp. Wallaceville Swamp near outlet $\left(41^{\circ} 08^{\prime} \mathrm{S}\right.$ Lat, $175^{\circ}$ 05' E Long), N161/618404. Coll. by W. F. Harris.

\section{Dunedin series}

\section{South Island}

Samples, except for NZ-341, from test bores on New Dental School Site NZ-337 and 338 in postglacial alluvium. NZ-339 and 340 in soliflual debris of Otira Glaciation. NZ-341 an interstadial (weak soil and vegetation) in loess and soliflual debris terrace. NZ-342 and 343 from thin alluvium interbedded with loess. Series gives a sequence of climatic events of the last glaciation. Coll. by J. D. Raeside, N. Z. Soil Bur.

\section{NZ-337.}

$$
\begin{array}{r}
1130 \pm 60 \\
\text { A.D. } 820
\end{array}
$$

Wood from outside of $\log 60 \mathrm{in}$. circumference charred charcoal at one end. Depth 18 in. in silty clay alluvium $\left(45^{\circ} 52^{\prime} \mathrm{S}\right.$ Lat, $170^{\circ} 30^{\prime} \mathrm{E}$ Long $)$, S164/157724.

NZ-338.

$$
1970 \pm 70
$$$$
20 \text { в.c. }
$$

Small tree with depth $4 \mathrm{ft} 6 \mathrm{in}$. in alluvial clay, within $8 \mathrm{ft}$ layer of silty clay derived from loess and basalt.

\section{NZ-339.}

$$
\begin{gathered}
11,500 \pm 170 \\
9550 \text { в.c. }
\end{gathered}
$$

Wood Hoheria from depth $8 \mathrm{ft}$, near base of stony gravel interbedded with silty clay derived from loess and basalt.

NZ-340.

$11,900 \pm 200$ 9950 в.c.

Wood from depth $8 \mathrm{ft} 6 \mathrm{in} .6 \mathrm{in}$. below top of gravelly horizon interbedded with silty clay derived from loess and basalt.

\section{NZ-341. New Kaikorai Valley School}

$31,300 \pm 900$

29,350 в.c.

Leaves and twigs $22 \mathrm{ft}$ below $15 \mathrm{ft}$ terrace, at top of stony bouldery 
horizon, with matrix of silty clay $\left(45^{\circ} 53^{\prime} \mathrm{S}\right.$ Lat, $170^{\circ} 28^{\prime} \mathrm{E}$ Long), S164/ 122277.

NZ-342.

$41,000 \pm 2300$

39,050 в.с.

Small tree from $5 \mathrm{ft} 6 \mathrm{in}$. below surface from near base of clay loam.

NZ-343.

$>39,000$

Wood from $3 \mathrm{ft}$ below surface in silty clay horizon $\left(45^{\circ} 52^{\prime} \mathrm{S}\right.$ Lat, $170^{\circ}$ $30^{\prime}$ E Long).

\section{Fiordland series}

A pollen profile in peat sequence shows strongly dominant conifer pollen at base changing to strongly beech pollen dominant above. At first Nothofagus Menziesii increases rapidly followed by greater increase of other species, apparently $N$ fusca and finally $N$ Solanderi var. cliffortioides, implying change from warm to cooler (and drier?) climate. Pollen analysis by W. F. Harris. Swamp on saddle E of Lake Monk, Fiordland $\left(40^{\circ} 00^{\prime} \mathrm{S}\right.$ Lat, $166^{\circ} 59^{\prime} \mathrm{E}$ Long). Subm. by W. F. Harris.

NZ-344. 9 in.

A.D. 1150

$800 \pm 60$

Peat, depth 9 in., $N$ Solanderi var. cliffortioides very strongly dominant.

NZ-345. 12 in.

Peat depth 12 in. Beech dominant.

NZ-346. 12-16 in.

Peat depth 12 to 16 in. Conifers codominant with beech.

NZ-347. 16-19 in.

Peat depth 16 to 19 in. Conifers dominant.

$$
1680 \pm 60
$$
A.D. 270

$$
\begin{aligned}
& 1810 \pm 65 \\
& \text { A.D. } 140
\end{aligned}
$$

$5610 \pm 90$

\section{Australia}

\section{NZ-348. Gormanstown, Tasmania}

Wood-bearing bed abuts against and is older than steeply dipping till. Gormanstown, near Queenstown, Western Tasmania $\left(42^{\circ} 04^{\prime} \mathrm{S}\right.$ Lat, $145^{\circ} 35^{\prime}$ E Long). Coll. by H. A. Bartlett. Univ. of Tasmania (Ahmad, Bartlett and Green, 1959).

\section{NZ-349. King Island, Tasmania}

Nothofagus driftwood, in alluvium $4 \mathrm{ft}$ above sealevel, overlain by deeply leached dune sand of the Old Dune system. Associated flora suggests slightly cooler or wetter climate than the present. Shorelines at $26 \mathrm{ft}$ and $45 \mathrm{ft}$ are as- 
signed to the last interglacial $\left(41^{\circ} 00^{\prime} \mathrm{S}\right.$ Lat, $144^{\circ} 09^{\prime} \mathrm{E}$ Long $)$. Coll. by J. N. Jennings, Australian Nat. Univ., Canberra.

Borneo

\section{NZ-350. Brunei Town, Borneo}

Wood, from youngest of several raised terraces. Muara Rd. $3.5 \mathrm{mi}$ from Brunei Town $\left(04^{\circ} 58^{\prime}\right.$ N Lat, $114^{\circ} 58^{\prime}$ E Long). Coll. by G. E. Wilford, Geol. Surv., Kuching Sarawak.

NZ-351. Brunei Town, Borneo

Wood from horizon similar to NZ-350, Muara Rd. $2.5 \mathrm{mi}$ from Brunei Town (04. $57^{\prime} \mathrm{N}$ Lat, $114^{\circ} 58^{\prime} \mathrm{E}$ Long).

\section{NZ-352. Lawas, Borneo}

$1950 \pm 75$

Dry fibrous peat from base of peat $10 \mathrm{ft}$ thick overlying estuarine sediments. Lawas, Fifth Div. Sarawak (04, $55^{\prime} \mathrm{N}$ Lat, $115^{\circ} 25^{\prime} \mathrm{E}$ Long).

\section{NZ-353. Sipangao Island, Borneo}

$28,000 \pm 600$

26,050 в.c.

Oyster shell from raised bench, $21 \mathrm{ft}$ above high tide, $\mathrm{N}$ coast of Sipangao Island $8 \mathrm{mi} \mathrm{S}$ of Semporna, North Borneo (04. $22^{\prime} \mathrm{N}$ Lat, $118^{\circ} 36^{\prime} \mathrm{E}$ Long). Coll. by H. J. C. Kirk, Geol. Surv., North Borneo. Comment: shell slightly recrystallized. Date is therefore a younger limit to the formation of the reef and the volcanic rocks on which it is built.

\section{ARCHAEOLOGY}

New Zealand

\section{Coromandel Peninsula series}

NZ-355 and 35625 yd N, 35950 yd N, 35860 yd N, 35770 yd N of stream cut gap in dunes, Moa-hunter site, Sarahs Gully, Coromandel Peninsula ( $36^{\circ} 42^{\prime}$ S Lat, $175^{\circ} 47^{\prime}$ E Long), N40/283799. All samples coll. by J. Golson, Auckland Univ.

\section{NZ-354. Mahinapua Bay}

$640 \pm 50$

\section{A.D. 1310}

Charcoal, Level 4C Squares 3, 4, 5, with shell, moa egg shell and archaic (moa hunter) culture $4 \mathrm{ft} 6$ in. below surface. Mahinapua Bay, $150 \mathrm{yd} N$ of mouth of Otama Creek ( $36^{\circ} 43^{\prime} \mathrm{S}$ Lat, $175^{\circ} 48^{\prime} \mathrm{E}$ Long), N40/287776.

\section{NZ-355. Level 4}

$\mathbf{6 0 0} \pm 50$

\section{A.D. 1350}

Charcoal, Level 4. Area D Square F7. Thin, rich Moa hunter occupation $4 \mathrm{ft}$ below blown sand of surface on terrace.

NZ-356. Level 3

Charcoal, from oven in culture Level 3 Area A Square G9, 1 ft 9 in. below surface. Dates an upper level in series of archaic layers. 
NZ-357. Level 3A

Charcoal, from culture Level 3A Area B square G15 and 1615 in. deep oven with moa pelvis on terrace.

NZ-358. Level 8

Wood from dwelling post culture Level 8 Posthole D Area A, dates building of intermediate archaic sequence.

\section{NZ-359. Level 9}

$650 \pm 50$

A.D. 1300

Charcoal, from culture Level 9 Area A Square H 11, 4 ft 3 in. below surface. From refuse pit representing earliest archaic occupation.

\section{Western Samoa}

\section{NZ-360. Safata, W Samoa}

$\mathbf{2 4 0} \pm \mathbf{5 0}$

\section{A.D. 1710}

Charcoal from hearths on $\mathrm{W}$ side of cave, 250 yds from entrance. Dist. of Safata $3 \mathrm{mi}$ inland from Sanapu village, Samoa. Seuao lava tunnel $\left(13^{\circ} 59^{\prime} \mathrm{S}\right.$ Lat, $171^{\circ} 53^{\prime} \mathrm{W}$ Long). Coll. by J. Golson. Comment: occupation more recent than the traditional one during the Tongan invasion 19 generations ago.

\section{Vailele Plantation series, W Samoa}

House mound $\mathrm{W}$ of new abattoirs. Vailele Plantation near Apia $\left(13^{\circ} 51^{\prime}\right.$ S Lat, $171^{\circ} 43^{\prime}$ W Long). Coll. by J. Golson.

\section{NZ-361. Layer 5, upper}

$$
1880 \pm 60
$$

Charcoal, from culture Layer 5, top half, under house mound. Layer contains first pottery found in Samoa.

\section{NZ-362. Layer 5, lower}

$1850 \pm 50$

Bottom half layer $5,5 \mathrm{ft}$ below surface of mound.

\section{NZ-363. Below Layer 5}

$$
\text { A.D. } 100
$$

Immediately below layer 5,6 to $6.5 \mathrm{ft}$ below mound surface in a pit, associated with pottery.

\section{Fromms Landing series, $\mathbf{S}$ Australia}

Fromms Landing, Lower Murray River, S Australia (34. $46^{\prime} \mathrm{S}$ Lat, $139^{\circ}$ $37^{\prime}$ E Long). Coll. by D. J. Mulvaney, Univ. of Melbourne.

\section{NZ-364. Basal occupation}

$$
\begin{aligned}
& 4800 \pm 100 \\
& 2850 \text { B.C. }
\end{aligned}
$$

Unio shells, from $4 \mathrm{in}$. band near lowest level of human occupation at this site. Overlain by levels containing cresentic microliths and pirri points. Gives maximum age for the overlying artifacts.

\section{NZ-365. Fromms Landing, 4 ft depth}

Charcoal, from layer of intense hearth occupation at a depth of $4 \mathrm{ft}$ from 
surface, marking a beginning of deterioration in production of techniques of stone and bone implements.

\section{NZ-366. Keilor Quarry, Melbourne}

$$
\begin{aligned}
& 15,000 \pm 1500 \\
& 13,050 \text { в.c. }
\end{aligned}
$$

Charcoal from carbonaceous lens $6 \mathrm{ft} 9 \mathrm{in}$. stratigraphically above NZ-207 $(18,000 \pm 500 \mathrm{yr})$ and at the same site, just below diastem. At another site charcoal from $2 \mathrm{ft} 6 \mathrm{in}$. above diastem gave $8500 \pm 250$ (W-169, USGS II). Keilor Cranium Quarry in Keilor Terrace; where Dry Creek enters Maribyrnong River $1 \mathrm{mi} \mathrm{N}$ of Keilor near Melbourne $\left(37^{\circ} 52^{\prime} \mathrm{S}\right.$ Lat, $144^{\circ} 50^{\prime} \mathrm{E}$ Long). Coll. by E. D. Gill, Natl. Mus. of Victoria. Comment: Keilor Cranium came from at or near this stratigraphic level. Sample is combined carbon and carbonate, date represents order of magnitude only.

\section{NZ-367. Glen Aire, Victoria}

$$
370 \pm 45
$$

Charcoal from depth of $5 \mathrm{ft} 9 \mathrm{in}$. to $6 \mathrm{ft}$ in Layer 4, the lowest layer to produce bone points similar to those collected from Warrnambool by E. D. Gill and dated (C-601) as $538 \pm 200$ yr. Rock shelter at Glen Aire, Cape Otway, Victoria ( $38^{\circ} 46^{\prime} \mathrm{S}$ Lat, $143^{\circ} 20^{\prime} \mathrm{E}$ Long). Coll. by D. J. Mulvaney.

\section{NZ-368. Rub'al Khali, Saudi Arabia}

$5090 \pm 200$

\section{0 в.c.}

Charcoal associated with Neolithic arrowheads from a camp site $\mathrm{E}$ of Jiladah, Rub'al Khali, Saudi Arabia ( $18^{\circ} 48^{\prime} \mathrm{N}$ Lat, $50^{\circ} 16^{\prime} \mathrm{E}$ Long). Coll. by B. Beverley; subm. by H. Field. Comment: Neolithic hunters apparently occupied this region at the time of the civilizations in Egypt, Mesopotamia.

\section{NZ-369. Cabeza, Peru}

\section{South America}

Fur robe that covered adult human skeleton, of prehorticultural settlement. Cemetary of Cabeza, Largu Peninsula, Peru ( $13^{\circ} 52^{\prime} \mathrm{S}$ Lat, $16^{\circ} 16^{\prime} \mathrm{W}$ Long). Coll. by F. Engel, Abancay 377, Lima, Peru.

\section{VOLCANOLOGY}

New Zealand

\section{NZ-370. Mayor Island}

$8390 \pm 135$

6440 в.c.

Wood, in pumice breccia, of first eruptive phase that formed main cone of Mayor Island. From cliff section in crater wall of main cone behind Te Paritu Lake ( $37^{\circ} 18^{\prime} \mathrm{S}$ Lat, $176^{\circ} 17^{\prime}$ E Long). Coll. by R. N. Brothers.

\section{NZ-371. Upper Waipa}

A.D. 1565

$385 \pm \mathbf{5 0}$

Charcoal from beds of alluvial pumice sand and conglomerate. Upper Waipa, road cut, immediately $\mathrm{E}$ of bridge over Waipa River 0.5 mile downstream of Okurawhanga Stream mouth ( $38^{\circ} 27^{\prime} \mathrm{S}$ Lat, $175^{\circ} 26^{\prime} \mathrm{E}$ Long), 
N83/930672. Coll. by D. Kear. Comment: although of similar appearance to the Taupo pumice alluvium NZ-1, 3, 4, etc. $(1800 \mathrm{yr})$, sample dates either a younger eruption or a pre-European fire that led to massive alluviation.

\section{NZ-372. Wellington City}

$8020 \pm 130$

6070 в.c.

Wood and roots from carbonaceous alluvial muds $18 \mathrm{in}$. above a pumiceous ash bed 3 in. thick. Sewage tunnel $90 \mathrm{ft} \mathrm{W}$ of corner Drummond St. and Adelaide Rd. $0.5 \mathrm{mi}$ from pre-European shoreline $\left(41^{\circ} 18^{\prime} \mathrm{S}\right.$ Lat, $174^{\circ} 47^{\prime} \mathrm{E}$ Long), N164/339196. Coll. by A. C. Beck and N. de B. Hornibrook, N. Z. Geol. Surv. Comment: date is minimum age for a violent rhyolitic eruption in the central volcanic district, some $180 \mathrm{mi} \mathrm{N}$. Pollen analysis (D. J. McIntyre) gives a Dacrydium cupressinum-dominant-flora, of warm postglacial type.

\section{NZ-373. Wharekauri, Chatham Islands}

$$
\begin{aligned}
& 39,600 \pm 2000 \\
& 37,650 \text { в.c. }
\end{aligned}
$$

Moorland peat from beneath $4 \mathrm{in}$. rhyolitic ash layer $12 \mathrm{in}$. below top of peat. In cutting on W side of Waitangi-Te Roto Rd. 8 chain S of Wharekauri turnoff $\left(45^{\circ} 50^{\prime} \mathrm{S}\right.$ Lat, $176^{\circ} 28^{\prime}$ W Long). Coll. by A. R. Mutch, N. Z. Geol. Surv. Comment: date places older limit on ash and violent rhyolitic eruption.

\section{NZ-374. Gataivai Village, W Samoa}

$$
\text { A.D. } 1190
$$

$\mathbf{7 6 0} \pm 50$

Coral sand on $15 \mathrm{ft}$ bench cut in Puapua basalt to $200 \mathrm{ft}$ inland of present cliff. Base of old sea cliff Gataivai, Savaii Island $\left(13^{\circ} 40^{\prime} \mathrm{S}\right.$ Lat, $172^{\circ} 24^{\prime} \mathrm{W}$ Long). Coll. by B. L. Wood, N. Z. Geol. Surv.

\section{NZ-375. Gataivai Village, W Samoa}

$715 \pm 50$

Coral fragments in gritty basalt ash, seaward edge of cliff. Gataivai Village, Savaii Island ( $13^{\circ} 40^{\prime}$ S Lat, $172^{\circ} 24^{\prime}$ W Long). Coll. by B. L. Wood and D. Kear. Comment: samples NZ-374 and 375 place limits on the Puapua basalt and give a rate of deformation by uplift along the central Savaii Island axis (Wood and Kear 1959).

\section{NZ-376. Puapua Village, W Samoa}

$$
\begin{aligned}
& 1850 \pm 80 \\
& \text { A.D. } 100
\end{aligned}
$$

Coral sand $5 \mathrm{ft}$ above present beach, overlain by Puapua Basalt, NW end of Puapua Village, Savaii Island ( $13^{\circ} 35^{\prime} \mathrm{S}$ Lat, $172^{\circ} 14^{\prime} \mathrm{W}$ Long). Coll. by B. L. Wood and D. Kear. Comment: dates a sealevel higher than the present and places an older limit on the Puapua Basalt.

\section{NZ-377. Cape Tapaga, W Samoa}

$$
1915 \pm 65
$$

Coral fragments from slightly cemented $6 \mathrm{in}$. bed, interbedded with Vini Tuff. Upolo Island, inland side of hillock on Cape Tapaga $\left(14^{\circ} 03^{\prime} \mathrm{S}\right.$ Lat, $171^{\circ} 25^{\prime}$ W Long). Coll. by B. L. Wood and D. Kear.

NZ-378. St Lucia, West Indies

Charred wood from volcanic ash that is among the latest within the Morne 
Gimie-Soufriere Caldera complex. Road cut near end of Ford St. Jacques upper road $1.5 \mathrm{mi} \mathrm{S}$ of summit of Morne Gimie-Soufriere area. St Lucia $\left(15^{\circ}\right.$ $51^{\prime} \mathrm{N}$ Lat, $61^{\circ} 10^{\prime} \mathrm{W}$ Long). Coll. by P. Martin-Kaye Ministry of Nat. Resources and Agriculture, Port of Spain, Trinidad.

NZ-379. Dominica, West Indies

Charred wood from pumice tuffs that were among the last products from the Microtrin-Trois Pitons center. Small excavation in pumiceous tuffs below hairpin bend in last inland local road near Roseau, behind Woodbridge Bay, Dominica ( $15^{\circ} 18^{\prime} \mathrm{N}$ Lat, $61^{\circ} 23^{\prime} \mathrm{W}$ Long). Coll. by P. Martin-Kaye.

\section{MISCELLANEOUS}

\section{New Zealand}

NZ-380. Hokianga, Auckland

Kauri (Agathis australis) log. Hokianga County, $\mathrm{N}$ Auckland. In road cut NW side No. 2 Road Omahuta State Forest ( $35^{\circ} 13^{\prime}$ S Lat, $173^{\circ} 39^{\prime}$ E Long), N10/161568. Coll. by C. F. Sutherland, N. Z. Soil Bur.

\section{Australia}

\section{NZ-381. Orroroo, S Australia}

Diprotodon Molar. 4 mi NE of Yalpara Station Homestead near Orroroo South Australia ( $32^{\circ} 30^{\prime} \mathrm{S}$ Lat, $138^{\circ} 55^{\prime}$ E Long), Coll. by S. Australian Mus., Lake Callabona, field party 1955. Note: surface scraped and washed in dilute HCl. Comment: sample checks significance of NZ-206, $6700 \pm 250$ as against NZ-205 >40,000.

Date lists:

\section{REFERENCES}

New Zealand I Fergusson and Rafter, 1953

New Zealand II Fergusson and Rafter, 1955

New Zealand III Fergusson and Rafter, 1957

New Zealand IV Fergusson and Rafter, 1959

New Zealand V Grant-Taylor and Rafter, 1962

USGS II $\quad$ Rubin and Suess, 1955

Ahmad, N., Bartlett, H. A., Green, D. H., 1959, The Glaciation of the King Valley, Western Tasmania: Roy. Soc. Tasm. Pap. 93: 11-6.

Baumgart, I. L., 1954, Some ash showers of the central North Island: New Zealand Jour. Sci. and Technology, sec. B, v. 35, p. 456-467.

Brodie, J. W., 1957, Late pleistocene beds, Wellington Penninsula: New Zealand Jour. Sci. and Technology, sec. B, v. 38, p. 623-643.

Brothers, R. N., and Golson, J., 1959, Geological and archaeological interpretation of a section in Rangitoto ash on Motutapu Island, Auckland: New Zealand Jour. Geol. and Geophysics, v. 2, p. 569-577.

Churchill, D. M., 1960, Late quaternary changes in the vegetation of Rottnest Island: Western Australian Naturalist, v. 7, p. 160-166.

Fergusson, G. J., 1954, The uses of carbondioxide filled proportional counters for radiocarbon dating: Nucleonics, v. 13, no. 1, p. 18-23.

1958, Reduction of atmospheric radiocarbon concentration by fossil fuel carbon dioxide and the mean life of carbon dioxide in the atmosphere: Australia Proc. Roy. Soc., v. 243 , p. 561-574. 
Fergusson, G. J., and Rafter, T. A., 1953, New Zealand ${ }^{14} \mathrm{C}$ age measurements I: New Zealand Jour. Sci. and Technology, sec. B, v. 35, p. 127-128.

1955, New Zealand ${ }^{14} \mathrm{C}$ age measurements II: New Zealand Jour. Sci. and

Technology sec. D, v. 36, p. 371-374.

1957, New Zealand ${ }^{14} \mathrm{C}$ age measurements III: New Zealand Jour. Sci. and Technology sec. B, v. 38, p. 732-749.

1959, New Zealand ${ }^{14} \mathrm{C}$ age measurements IV: New Zealand Jour. Geol. and Geophysics, v. 2, p. 208-241.

Field, H., 1959, Anthropological reconnaissance in West Pakistan 1955: Peabody Mus. Papers, v. 52 , p. $1-332$.

1960, Carbon 14 date for a neolithic site in the Rub 'al Khali: Man., v. 60, p. 172 (article 214).

Grant-Taylor, T. L., and Rafter, T. A., 1962, New Zealand radiocarbon age measurements V: New Zealand Jour. Geol. and Geophysics, v. 5, p. 331-359.

Jansen, H. S., 1962, Comparison between ring-dates and ${ }^{14} \mathrm{C}$ dates in a New Zealand Kauri Tree: New Zealand Jour. Sci., v. 5, p. 74.

Jennings, J. N., 1959, The coastal geomorphology of King Island: Recs. Queen Victoria Museum Launceston N. S., v. 11, p. 1-39.

1961, Sea level changes in King Island, Bass Strait: Zeitschrift für Geomorphologie Supp., v. 3, p. 80-84.

Kear, D., and Wood, B. L., 1959, The geology and hydrology of Western Samoa: New Zealand Geol. Surv. Bull., new series 63.

Libby, W. F., 1951, Radiocarbon dates, II: Science, v. 114, p. 291.

McCallum, G. J., 1955, Evaluation of the accuracy of New Zealand radiocarbon dating results: New Zealand Jour. Sci. and Technology, sec. B, v. 37, p. 370-381.

McKellar, I. C., 1960, Pleistocene deposits of the upper Clutha Valley Otage, New Zealand: Jour. Geol. and Geophysics, v. 3, p. 432-460.

Mulvaney, D. J. 1959, Dating of Australian prehistory: Nature, v. 184, no. 4690, p. 918

Pantin, H. M., 1957, Fossiliferous concretions from the shelf south-east of Cape Campbell, New Zealand: New Zealand Jour. Sci. and Technology, v. 38, p. 781-791.

Rafter, T. A., 1953, The preparation of carbon for $C^{14}$ age measurements: New Zealand Jour. Sci. and Technology, sec. B, v. 35, p. 64-89.

1955a, Carbondioxide as a substitute for solid carbon in ${ }^{14} \mathrm{C}$ age measurements: New Zealand Jour. Sci. and Technology, sec. B, v. 36, p. 363-370.

$1955 \mathrm{~b},{ }^{14} \mathrm{C}$ variations in Nature and the effect on radiocarbon dating: New Zealand Jour. Sci. and Technology, sec. B, v. 37, p. 20-38.

Rubin, Meyer, and Suess, H. E., 1955, U. S. Geological Survey radiocarbon dates II: Science, v. 121, p. 488.

Schofield, J. C., 1960a, Sea level fluctuations during the past four thousand years: Nature, v. 185 , no. 4716 , p. 836.

$1960 \mathrm{~b}$, Sea level fluctuations during the last 400 years as recorded by a chenier plain, Firth of Thames, New Zealand: New Zealand Jour. of Geol. and Geophysics, v. 3 , p. 467 .

Searle, E. J., 1959a, Pleistocene and recent studies of Waitemata Harbour, part 2, North Shore and Shoal Bay: New Zealand Jour. of Geol. and Geophysics, v. 2, p. 95-107. 1959b. The volcanoes of Ihumatao and Mangere, Auckland: New Zealand Jour. Geol. and Geophysics, v. 2, p. 870-888.

Suggate, R. P., 1958a, Geology of the Harper and Avoca River Valleys Mid Canterbury, New Zealand: New Zealand Jour. Geol. and Geophysics, v. 1, p. 31-46.

1958b, Late quaternary deposits of the Christchurch Metropolitan area: New Zealand Jour. Geol. and Geophysics, v. 1, p. 103-122.

Te Punga, M. T., 1958, Evidence for a low sea level 9900 years ago: New Zealand Jour. Geol, and Geophysics, y. 1, p. 92-94.

Vries, Hessel de, 1958, Variation in concentration of radiocarbon with time and location on earth: Koninkl. Nederlandse Akad. Detensch. Proc. Ser. B, v. 61, no. 2, p. 1-9.

Willis, E. H., Tauber, H., Münnich, K. O., 1960, The variations in atmospheric radiocarbon, Am. Jour. Sci. Radioc. Supp., v. 2, p. 1-4. 